\title{
Megabase-scale presence-absence variation with Tripsacum origin was under selection during maize domestication and adaptation
}

Yumin Huang ${ }^{1 \dagger}$, Wei Huang ${ }^{1+}$, Zhuang Meng ${ }^{2}$, Guilherme Tomaz Braz ${ }^{3}$, Yunfei Li ${ }^{1}$, Kai Wang ${ }^{2}$, Hai Wang ${ }^{1}$, Jinsheng Lai ${ }^{1}$, Jiming Jiang ${ }^{3}$, Zhaobin Dong ${ }^{1 *}$ (D) and Weiwei Jin $^{1 *}$

\author{
*Correspondence: zbdong@cau. \\ edu.cn; weiweijin@cau.edu.cn \\ †Yumin Huang and Wei Huang \\ contributed equally to this work. \\ ${ }^{1}$ State Key Laboratory of Plant \\ Physiology and Biochemistry, \\ National Maize Improvement \\ Center, Key Laboratory of Crop \\ Heterosis and Utilization (MOE), \\ Joint International Research \\ Laboratory of Crop Molecular \\ Breeding (MOE), China Agricultural \\ University, Beijing 100193, China \\ Full list of author information is \\ available at the end of the article
}

\begin{abstract}
Background: Structural variants (SVs) significantly drive genome diversity and environmental adaptation for diverse species. Unlike the prevalent small SVs (< kilobase-scale) in higher eukaryotes, large-size SVs rarely exist in the genome, but they function as one of the key evolutionary forces for speciation and adaptation.

Results: In this study, we discover and characterize several megabase-scale presence-absence variations (PAVS) in the maize genome. Surprisingly, we identify a 3.2 Mb PAV fragment that shows high integrity and is present as complete presence or absence in the natural diversity panel. This PAV is embedded within the nucleolus organizer region (NOR), where the suppressed recombination is found to maintain the PAV against the evolutionary variation. Interestingly, by analyzing the sequence of this PAV, we not only reveal the domestication trace from teosinte to modern maize, but also the footprints of its origin from Tripsacum, shedding light on a previously unknown contribution from Tripsacum to the speciation of Zea species. The functional consequence of the Tripsacum segment migration is also investigated, and environmental fitness conferred by the PAV may explain the whole segment as a selection target during maize domestication and improvement.
\end{abstract}

Conclusions: These findings provide a novel perspective that Tripsacum contributes to Zea speciation, and also instantiate a strategy for evolutionary and functional analysis of the "fossil" structure variations during genome evolution and speciation.

Keywords: Maize, Tripsacum, Presence-absence variation, NOR, Domestication

\section{Introduction}

A striking range of natural genetic diversity has been revealed at the level of SNPs, indel polymorphisms (IDPs), and structural variation [1-4]. Structural variation involves an order of magnitude higher base pairs relative to SNP, which is the largest source of genetic variation $[4,5]$. Structural variation mainly includes presence-

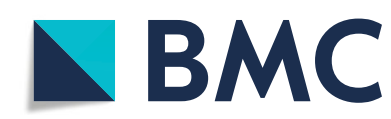

(c) The Author(s). 2021 Open Access This article is licensed under a Creative Commons Attribution 4.0 International License, which permits use, sharing, adaptation, distribution and reproduction in any medium or format, as long as you give appropriate credit to the original author(s) and the source, provide a link to the Creative Commons licence, and indicate if changes were made. The images or other third party material in this article are included in the article's Creative Commons licence, unless indicated otherwise in a credit line to the material. If material is not included in the article's Creative Commons licence and your intended use is not permitted by statutory regulation or exceeds the permitted use, you will need to obtain permission directly from the copyright holder. To view a copy of this licence, visit http://creativecommons.org/licenses/by/4.0/. The Creative Commons Public Domain Dedication waiver (http://creativecommons.org/publicdomain/zero/1.0/) applies to the data made available in this article, unless otherwise stated in a credit line to the data. 
absence variants (PAV), copy number variants $(\mathrm{CNV})$, inversions, translocations, and more complex rearrangement $[6,7]$. One extreme form of $\mathrm{CNV}$ is the presenceabsence variation (PAV), in which a particular sequence is present in some individuals while absent in others. The advance of long- and short-read sequencing technologies has significantly boosted the identification of PAVs in numerous species [8-10]. However, the prevalence of PAVs in the genome-wide context varies among species as well as individual samples. For example, PAVs have been prevalently identified in bacterial genomes since firstly found in the prokaryotes decades ago [11, 12], and the intraspecific PAVs in bacteria could count for over half of the genome [13]. In contrast, in eukaryotes particularly in higher animals including humans, PAVs were found often with a much smaller size scale, and they predominantly consist of intergenic sequences [14, 15]. Nevertheless, a growing number of eukaryotes, such as fungi, algae, and plant species, have been reported with large-size PAVs containing functional genes $[9,10,16-$ 19].

Compared to small PAVs, large-size PAVs bring instability of the genome, possibly resulting in their failure in the transmission to the subsequent generations [20]. Coding genes and/or regulatory elements within large PAVs may lead to deleterious or even lethal lesions for biological function or genome integrity, consequently showing vulnerability to evolution purification in the diversity population [16]. More importantly, even if large variations could confer elevated fitness and thus escape from sequence divergence and erosion, it is almost inevitable that they will gradually be rearranged into smaller-sized variations, as a result of intraspecific homologous recombination [21].

Multiple mechanisms have been proposed for the PAV formation in the genome, such as the copy/cut-paste mechanism by transposable elements and improperly paired meiotic recombination [22, 23]. These processes, however, antagonized by the genome repair mechanism, may not be effective to raise large-size PAVs. Instead, interspecific gene transfer and genome fractionation following duplication have been reported as the important ways to generate large PAVs [24, 25]. For example, bacteria can either incorporate exogenous DNA into their own genomes, or exchange genetic elements between individuals, leading to prevalent large-size PAVs [26]. In plant species, interspecific hybridization is ubiquitous during speciation [27, 28]. The interspecific hybridization generates large PAVs, likely due to the homologous exchange between the progenitor genomes [29-32]. It has been shown that large-size PAVs in several polyploidy crops were raised in the process of polyploidization and the subsequent genome fractionation [33]. Therefore, large PAVs could be an excellent system to infer novel insights for speciation and evolution. In fact, SVs play critical roles in understanding the genetic contributions of plant domestication history and phenotypic plasticity [34]. However, due to the current lack of evaluation of large-size PAVs in plants and their own rarity, it is still ambiguous if interspecific transfer or genome fractionation directly links to the large-size PAVs.

Maize (Zea mays ssp. mays) is an ancient tetraploid [35, 36]. The polyploidization, chromosome rearrangement, and the following genome fractionation attributed to the Zea speciation are believed to generate prevalent SVs including large PAVs [37, 38]. The maize genome is highly malleable, which can also be described by a surprisingly large amount of SV [39-41]. Indeed, nearly 4000 instances of CNV/PAV were observed in a diversity panel of maize and the wild ancestor teosinte lines [42], although their 
dynamics underlying speciation and the later domestication are still largely unknown. Modern maize was domesticated from teosinte (Zea mays ssp. parviglumis) and about 2 to $4 \%$ of all maize genes experienced artificial selection [43]. Along with that, significant introgression has also been reported with genomic fragments flowing from another teosinte sister species Zea mays ssp. mexicana [44, 45]. The potential gene flow from other Zea species that are interfertile with maize, such as Zea luxurians, possibly has also contributed to the maize genome evolution and domestication [46]. Outside the Zea genus, the closest relative is the genus Tripsacum [47], which had been notoriously proposed as the domestication origin for maize until the role of teosinte was confirmed [48]. Rare cases of natural and artificial Zea-Tripsacum hybrids have been identified, but the offspring are invariably sterile [48, 49]. Nevertheless, there has been little evidence to rule out the possibility that Tripsacum was interfertile with the Zea ancestor in ancient times. Investigation on the potential PAVs directly resulted from the interspecific introgression could be a promising strategy to sketch the process through Zea speciation to domestication.

An exceptional PAV at megabase scale has been revealed between two heterotic inbred lines B73 and Mo17 [50, 51]. The recent revolutionary sequencing strategy using single-molecule technologies has improved the quality of the de novo genomes of B73 and Mo17, providing extensive information on the genome-wide variations between these two inbreds $[3,52]$. However, the insights on the genomic and evolution signature, and the functional consequence of the largest PAVs that contains hundreds of genes in these regions, are still intriguing questions to be investigated. Here, we conducted comparative genomics analysis of B73 and Mo17 and documented a $3.2 \mathrm{Mb}$ PAV within the nucleolus organizer region (NOR) on chromosome 6 . The entire sequence of this PAV is largely intact in a diversity panel ranging from teosinte to maize, likely due to the suppressed recombination by NOR to counteract the evolution sequence erosion. A series evidence indicated that this PAV originated from Tripsacum, providing direct support for the contribution of Tripsacum to the Zea speciation. Lastly, we found this Tripsacum-originated PAV may be consistent with selection during maize domestication and improvement, by having potential function on environmental adaptation.

\section{Results}

Fine mapping of megabase-scale PAVs between maize inbreds B73 and Mo17

To capture large-size PAVs in the genome context of maize inbred lines, we compared the recent genome assembly of B73 and Mo17, which have been recently improved by single-molecule sequencing technique $[3,52]$. We aimed to isolate the large PAVs with sizes over $500 \mathrm{~kb}$ that are present in one inbred while absent in the other. Here we used a sliding-window method that was previously reported which divided one reference genome into 500-bp windows with a step size of $100 \mathrm{bp}$ and aligned to another genome; the windows that could not be aligned to the other genome were merged within $100 \mathrm{~kb}$ of the physical coordinates and then defined as a PAV cluster [3]. Three regions (designated as RegionA, RegionB, and RegionC hereinafter, with a size of $2.9 \mathrm{Mb}, 628 \mathrm{~kb}$, and $547 \mathrm{~kb}$, respectively) were found present only in B73, while two regions (designated as RegionD and RegionE with a size of $2.5 \mathrm{Mb}$ and $753 \mathrm{~kb}$, respectively) were found only 
in Mo17 (Additional file 2: Table S1); these results are consistent with the previous study [3].

Using the recent publicly available $\mathrm{Hi}-\mathrm{C}$ data for $\mathrm{B} 73$ chromatin architecture and the newly improved B73 AGPv4 genome as a reference [52, 53], we were able to validate the physical location and orientation of these three large PAVs in B73. Consistent with the genome-wide profiling that chromatin interaction matrix substantially matched with genome assembly, the two smaller PAVs, RegionB and RegionC, were verified based on the consistency between the Hi-C data and genome reference (Additional file 1: Figure S1a, b). However, an abnormal interaction was observed between $22 \mathrm{M}$ to 25 $\mathrm{M}$ on chromosome 6 based on AGPv4 genome, which maps to the exact location of the largest PAV RegionA (Additional file 1: Figure S1c), suggesting a putative assembly error in this region. Therefore, we revisited this scaffold and indeed found a Bionano map error. Eventually, an updated version of assembly for this region was rectified and designated as AGPv4.1, based on which the RegionA location was adjusted to the physical interval approximately between $13 \mathrm{M}$ and $16 \mathrm{M}$ on Chromosome 6 (Additional file 1: Figure S1d, e). As a consequence of the improved accuracy of AGPv4.1, the length of RegionA was determined to be $3.2 \mathrm{Mb}$, which was slightly expanded than the previous reports $[3,50]$. In total 70 protein-coding genes were annotated in this region (Additional file 2: Table S1).

We remapped Mo17 resequencing data [54] against the B73 genome reference and vice versa and observed nearly all the annotated genes $(12 / 13,6 / 8$, and 13/20) in RegionB, RegionC, and RegionE had the homologous alleles in the genome of the absence counterpart (CDS coverage $>80 \%$ ), suggesting these PAVs may be caused by translocation rather than deletion. In contrast, for both of the megabase PAVs, 65 out of 70 RegionA genes and 9 out of 10 RegionD genes were found completely missing in the Mo17 and B73 genome (CDS coverage <50\%) respectively, suggesting that gene sets contained in RegionA and RegionD are true presence-absence alleles.

We designed a FISH probe using single-copy oligos within the RegionA region. The oligo-FISH signals were detected in the expected region on Chromosome 6 in B73 (Fig. 1a); in contrast, no signals were detected in Mo17 (Fig. 1b), substantiating the absence of RegionA in Mo17. Consistently, the B73/Mo17 F1 hybrids showed FISH signal only on the B73 chromosomes while missed on the Mo17 allelic location (Fig. 1c).

PAVs RegionA and RegionD present in the diversity population as integrated elements

We next investigated the prevalence of these large PAVs in the maize natural variation population. A set of genome resequencing data was used for analysis, including 17 teosinte lines (2 Zea ssp. mexicana and 15 Zea ssp. parviglumis), 23 landraces, 60 maize inbreds [55], and 1 Zea luxurians line [56]. In addition, as sister genus related to Zea, 4 Tripsacum [57] and 9 sorghum lines [58] were also included (Additional file 2: Table $\mathrm{S} 2$ ). Through a 10-kb sliding window analysis with a step size of $1 \mathrm{~kb}$, all five large PAVs between B73 and Mo17 were characterized in the above-mentioned accessions (Fig. 1d, Additional file 1: Figure S2 and 3). B73 PAVs RegionB, RegionC, and Mo17 PAVs RegionE showed a continuous distribution of presence-bin proportion among the diversity lines (Additional file 1: Figure S2a,b and Additional file 1: Figure S3b), suggesting extensive divergence in these PAV regions. 


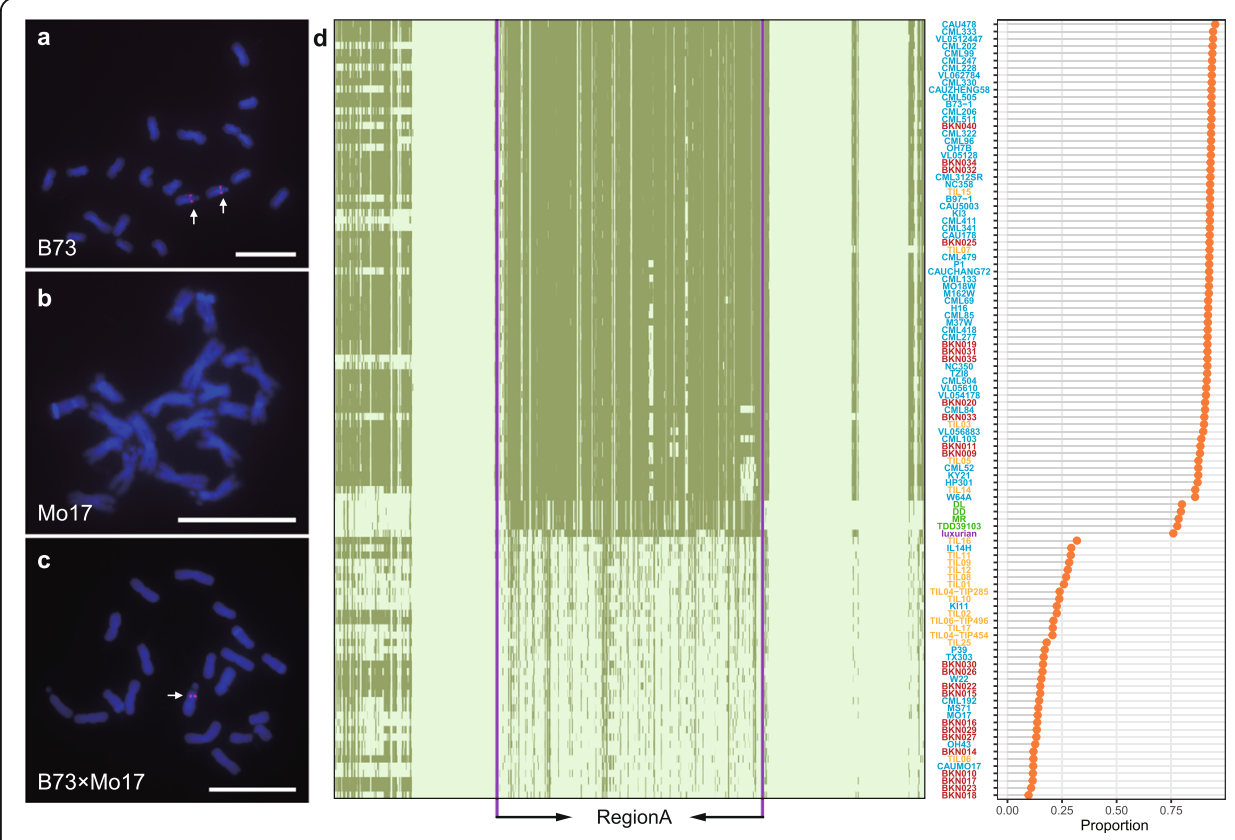

Fig. 1 Characterization of a megabase-scale PAV, RegionA, in diverse maize inbreds and their wild relatives. a-c Fluorescence in situ hybridization (FISH) of RegionA-specific oligo probes on root tip metaphase chromosomes of B73, Mo17, and their F1 hybrid. RegionA-specific signals (red) were observed on a pair of homologous chromosomes of B73 metaphase cells (a), while absent on Mo17 metaphase chromosomes (b). Only one foci signal can be detected on mitotic metaphase spreads of B73 $\times$ Mo17 F1 hybrid (c). Scale bars $=10 \mu \mathrm{m}$. $\mathbf{d}$ Left: PAV map of RegionA and its flanking regions ( $2 \mathrm{Mb}$ on each side) in diverse maize inbreds and their wild relatives. Each column represents a bin (dark green for presence bin, light green for absence bin), and each row represents a line (text color of green for Tripsacum; purple for Zea luxurians; yellow for teosintes; red for maize landraces; blue for maize inbreds). Right: The proportion of presence bins in RegionA for each line

In contrast, the two megabase-scale PAVs, RegionA of B73 and RegionD of Mo17, showed a surprisingly polarized pattern of genotype distribution: contrasting groups of lines with presence-bin proportion of either less than $30 \%$ or larger than $75 \%$; besides that none of the lines were found to contain an intermediate presence-bin proportion (Fig. 1d and Additional file 1: Figure S3a). In addition to the above genome resequencing data, the stable feature of both RegionA and RegionD was further confirmed by the syntenic comparison between multiple inbreds with de novo genome assembly including 26 high-quality NAM founders genome using PacBio long reads, a mate-pair strategy [59-61]. B73 RegionA showed well collinearity with the genome of PH207, B104, and 19 out of 24 other NAM founders besides B73 (Additional file 1: Figure S4), but no collinearity with Mo17, W22, and remaining NAM founders, consistent with the presence-bin proportion pattern shown in Fig. 1d. We further investigated the coding sequence coverage of RegionA genes by mapping HapMap2 resequencing reads to B73 reference genome, and the results showed that different from RegionB and RegionC, the majority of RegionA genes are nearly completely present or absent in diversity lines (Additional file 1: Figure S5a). In accordance with that, FISH experiment using RegionA-specific oligo probes showed signals in the RegionApresent inbreds while none detected in the absent lines (Additional file 1: Figure S5b-e). These results suggested an unexpected scenario that the majority of RegionA and RegionD appear to be present or absent as an integrated element among the diversity panel, only experiencing extremely low levels of internal sequence divergence within the region. 
RegionA infers an origination from Tripsacum

Given the integrated feature of RegionA and RegionD, we grouped the diversity accessions based on the presence or absence of the PAVs. RegionD appeared to be a relatively rare allele, present only in $10 \%$ of the accessions tested in this study, including two inbreds (Mo17 and Ms71) and several lines of landrace and teosinte. In contrast, the frequency of RegionA is much higher, as it is present in $66 \%$ of all the accessions tested, including most inbreds, several landraces, and teosinte lines (Fig. 1d). We also identified the RegionA genotypes in the diversity panel of maize, and the results showed that no RegionA heterozygotes were found in 100 HapMap2 lines, but 26 of 99 Meso- and South America lines were defined as RegionA heterozygotes (Additional file 1: Figure S6). Interestingly, 4 of 4 (100\%) Tripsacum and the one Zea luxurians contained major portions (76\% 80\%) of RegionA (Fig. 1d), suggesting the emergence of this element predated the divergence of Zea and Tripsacum from their common ancestor. Nevertheless, RegionA was not observed in the 9 sorghum lines (Additional file 1: Figure S2c), and no collinearity of RegionA was found in more distantly related species, including Setaria and rice (Additional file 1: Figure S4). Though we cannot rule out the possibility that RegionA may also be present in a more distant genus but undetectable due to the lack of a corresponding genome, our result to date indicates RegionA appears as a specific element for the Tripsacinae subtribe.

The Tripsacinae specific sequence of RegionA inspired us to trace the origin of this largest PAV. The long terminal repeat retrotransposon (LTR-RT) is the most abundant repeat type in the maize genome, and the divergences of LTR retrotransposons have been widely used to estimate the date of insertion for landmarking the evolution of flanking region [62]. Approximately 68\% of transposable elements (TE) were annotated as LTR-RTs in RegionA, a proportion similar to the genome-wide level (Additional file 1: Figure S7a,b). Nevertheless, the RegionA was found to consist of more unknown superfamily retrotransposons (RLX) compared to the average genome-wide level (Additional file 1: Figure S7c). Besides, almost 70\% of the LTR-RTs were annotated as nonmaize-family (Additional file 1: Figure S7d). By calculating the LTR insertion time in RegionA, we estimated that the oldest insertion event occurred approximately 1.74 Mya (Additional file 1: Figure S7e). This is earlier than the divergence of Zea from Tripsacum genus which was estimated no earlier than 1.2 million years ago [63]. This result suggested that RegionA, or at least parts of this element, can be traced back to the common ancestors of Tripsacum genus and Zea genus.

As types of LTR-RTs show various abundance in the genome, we examined the genome-wide abundance of the corresponding LTR-RTs types in RegionA (see "Methods") and found a major proportion of RegionA LTR-RTs represents the types with low genome-wide abundance (Additional file 1: Figure S7f). This result suggested that despite the ancient nature of this region, the containing LTR-RTs were predominantly trapped in the local region rather than expanding to the global genome. All the LTR retrotransposons in RegionA were then categorized by the abundance in the context of the Zea and Tripsacum genome background, respectively (Additional file 2: Table S3). The low abundance LTR retrotransposons relative to the B73 surprisingly shared an intensive identity with the highly abundant LTR retrotransposons in the Tripsacum genome (designated as Tripsacum-specific enriched LTR retrotransposons, or Tse-LTR-RTs hereafter) (Fig. 2a, i). To visualize the genomic distribution of the 
Tse-LTR-RTs between maize and Tripsacum, we developed probes from three selected Tse-LTR-RTs for FISH analysis. As expected, the Tse-LTR-RTs probes showed strong hybridization signals throughout the Tripsacum chromosomes, whereas no unambiguous signals were detected on maize chromosomes (Fig. 2b-g, Additional file 1: Figure S7g-i). Interestingly, a previous study has isolated several Tripsacum-specific retroelements that showed specific FISH hybridization in Tripsacum lineage but not in the Zea species [64]. We validated these Tripsacum-specific retroelements by BLAST search against the B73 genome and filtering with stringent parameters, and found all perfectly matched loci were located exclusively in RegionA (Additional file 2: Table S4), substantiating the enrichment of Tripsacum LTR-RTs in RegionA. Additionally, evidence from homologous coverage and genes also highlighted the major contribution from Tripsacum to this region (Fig. 2i).

Among the LTR retrotransposons in RegionA, the Tse-LTR-RTs were estimated to be older than the remaining retrotransposon counterparts, suggesting the Tripascumoriginated components may serve as the initial foundation for the later divergence of this region (Additional file 1: Figure S7j). This intrigued us to investigate whether RegionA was originally raised by a transfer from ancient Tripsacum, as interspecific transfer has been instanced to generate PAVs in plant genome [65]. Indeed, a treemix analysis identified signals for RegionA introgression from Tripsacum to Zea (Fig. 2h, Additional file 1: Figure S8a,b). This introgression from Tripsacum appeared unique only within RegionA, since the same analysis using the whole chromosome 6 as inputs only showed intraspecies gene flow among Zea genus whereas the introgression from Tripsacum was not detectable (Additional file 1: Figure S8c-e). The analysis for absolute sequence divergence $\left(d_{x y}\right)$ has been generally used as the benchmark for distinguishing recent gene flow from shared ancestral variation [66, 67]. We measured the $d_{x y}$ of RegionA between Tripsacum and Zea. Compared with the rest of the genome, RegionA showed a dramatically reduced $d_{x y}$ that visualized as a pattern of trough on the entire chromosome (Additional file 1: Figure S9a), and the $d_{x y}$ value of $60.5 \sim$ 72.2\% RegionA windows was below the cutoff of whole-genome bottom 5\% (Additional file 1: Figure S9b). This result shows a distinct signal of reduced divergence between Tripsacum and Zea at RegionA, which is likely due to the recent coalescence at this region led by introgression. We attributed the exclusive detection for Tripsacum origin to the characteristic integrity of RegionA, as the ancient flow in RegionA is still well preserved while the rest of the genome has been shuffled. These results proposed the segment transfer between the two sister species, arguing a possible direct contribution from Tripsacum in the speciation of Zea family.

\section{Suppressed recombination by $45 \mathrm{~S}$ rDNA contributed to maintaining the integrity of RegionA}

We wondered if RegionA and RegionD are located in recombination-suppressed regions, which may result in the intriguing integrity of these sequences in the diversity panel. Indeed, RegionD was found located in the pericentromeric region of Chromosome 6, characterized with a low rate of recombination [68].

RegionA, in contrast, is located on the distal end of Chromosome 6 short arm, far from the pericentromere; therefore, this region was generally supposed to have a high 


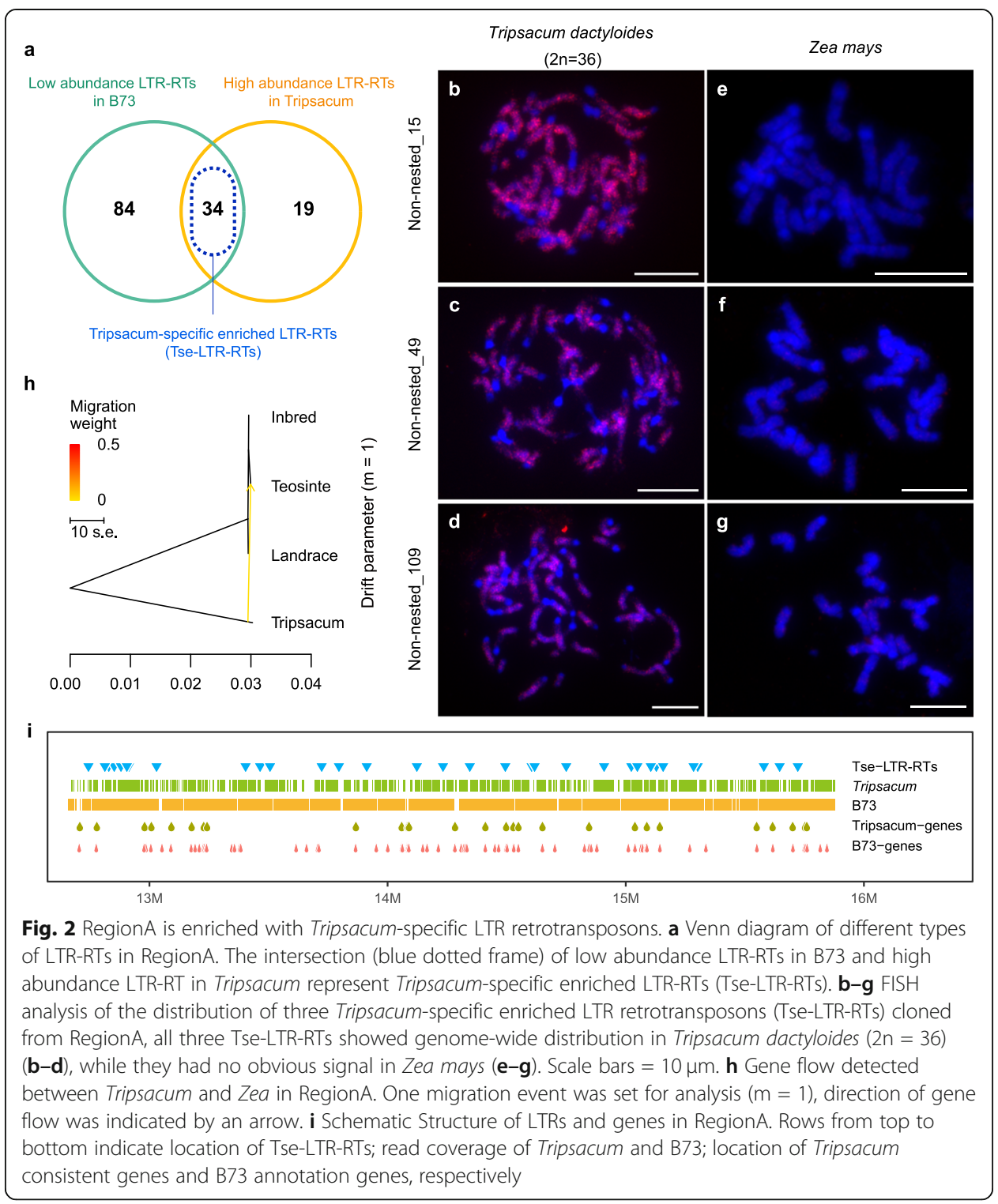

rate of recombination. However, the short arm of Chromosome 6 is known to carry the $45 \mathrm{~S}$ rDNA and a chromosomal "satellite", and it is also associated with a low level of crossovers (Fig. 3a) [68-70]. The low recombination rate in this area was believed to be caused by the nucleolus organizer region (NOR) located in this region [70, 71]. Due to the highly repetitive nature of the NOR, this region is poorly assembled. Even in our updated AGPv4.1, this region still contains numerous gaps (Additional file 1: Figure S1d). To determine the accurate location of RegionA relative to the NOR, FISH assay was performed in B73 and B73 $\times$ Mo17 hybrid meiotic pachytene using an oligonucleotide-based probe specific to RegionA (see "Methods") together with a 45S rDNA probe. The FISH results indicated that RegionA was embedded within the 45S rDNA arrays and was mapped toward the proximal end of NOR (Fig. 4a-f). Mapping $45 \mathrm{~S}$ rDNA sequence back to the AGPv4.1 genome also revealed the presence of $45 \mathrm{~S}$ rDNA sequences around the RegionA (Fig. 4g). Note that the schematic amount of $45 \mathrm{~S}$ rDNA was possibly underestimated due to the unfilled gaps. 


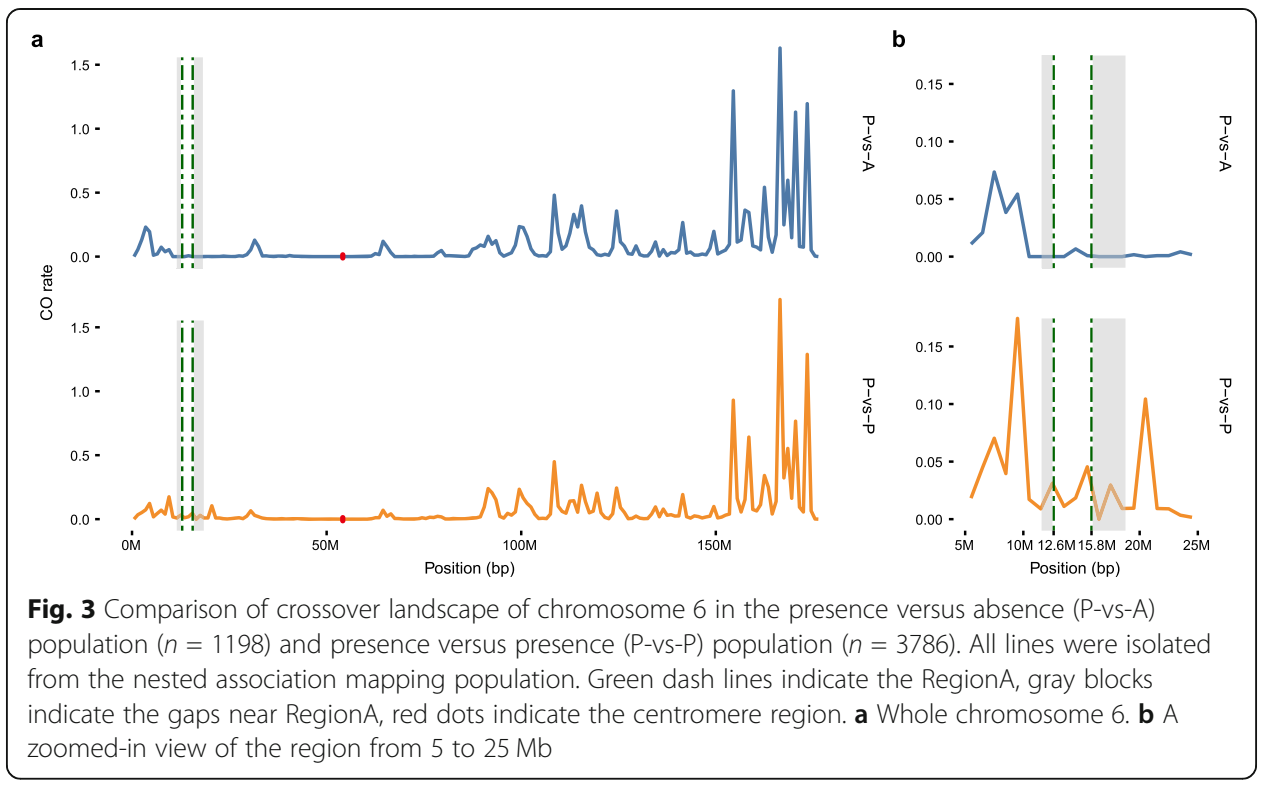

It has been widely acknowledged that structural variations, including PAVs, also strongly affect the frequency and distribution of recombination events in animals and plants [72, 73]. Despite the low recombination rate around the NOR, we noticed that the recombination rate was even more suppressed in the NAM RILs from RegionAabsence founder pairs, in contrast to that from all RegionA-presence founders (Fig. 3b). Therefore, the suppressed recombination, caused by a heterochromatic chromosomal environment as well as the structural variation itself, may play a major role, to maintain the intact status for megabase-scale RegionA and RegionD during million years of evolution.

\section{RegionA was under selection during maize domestication and improvement}

To obtain genetic insights into RegionA and domestication, we surveyed polymorphism and divergence at this region with different subgroups. Average overall $\pi$ (nucleotide diversity) and $\theta_{W}$ (population mutation rate) for teosinte, landrace, and inbred in RegionA are $0.00079,0.00066,0.00083$ and $0.00083,0.00079,0.00160$, respectively. They are all significantly lower than the level of whole chromosome 6 (Fig. 5a,b). Within RegionA, pre- and post-domestication lines showed little evident difference of nucleotide diversity (Fig. 5a), but a relatively lower level of population mutation rate was found in teosinte and landrace compared to inbred (Fig. 5b).

Since RegionA has been extremely conserved due to the suppressed recombination brought by NOR, we wonder whether this effect spreads to the peripheral area of NOR. Here we made the pairwise comparison of nucleotide diversity between RegionA and upstream or downstream flanking regions of NOR with different scales. We found that the $\pi$ value showed no difference between RegionA and upstream $100 \mathrm{~kb}$ and downstream $500 \mathrm{~kb}$ (Additional file 1: Figure S10a,b), while inside these two regions there was almost no difference between RegionA-presence lines and absence lines (Additional file 1: Figure S10c,d). 


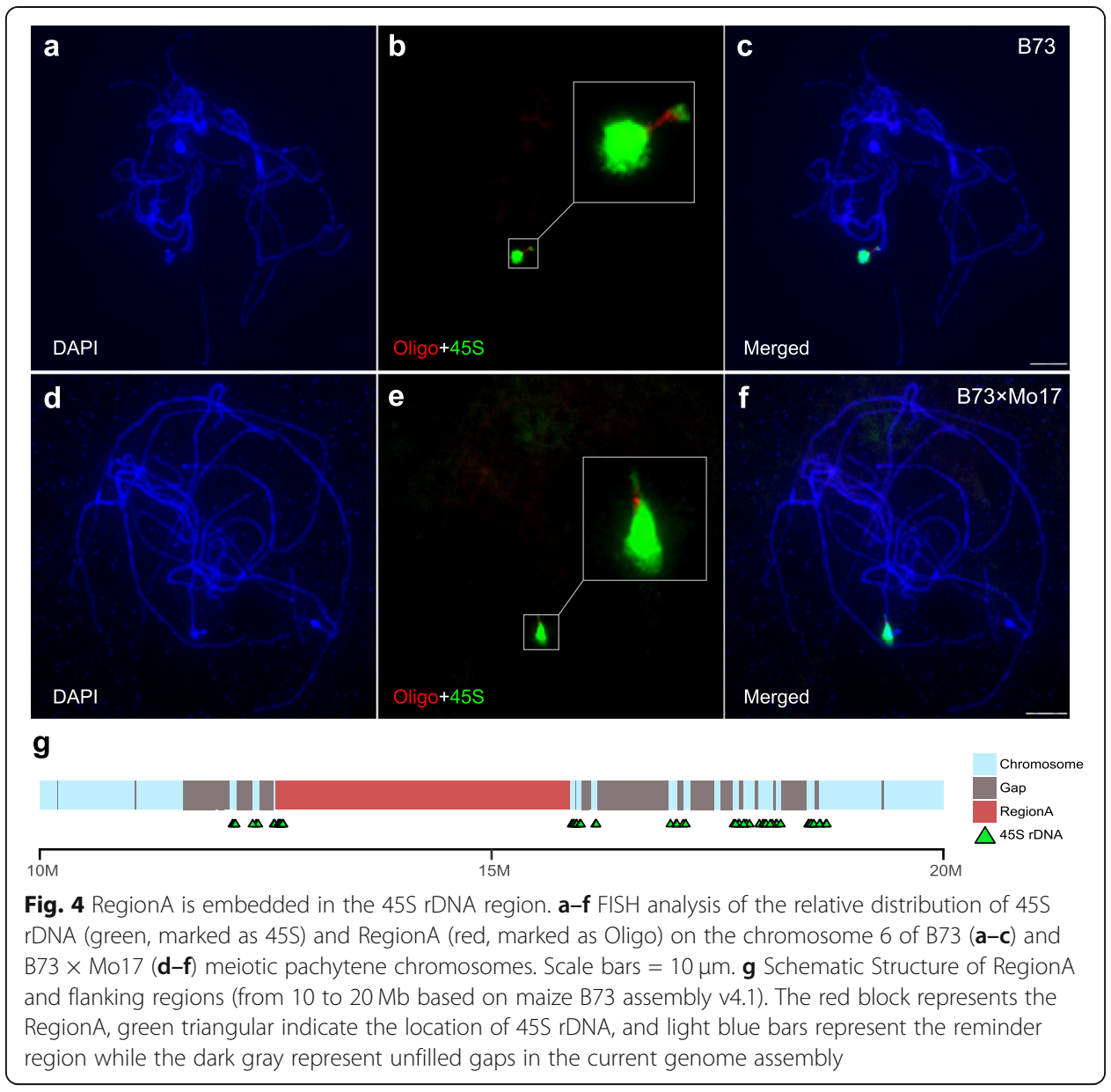

To further investigate the phylogenetic relationships among diversity panels in RegionA, we extracted the SNPs of the flanking region mentioned above for phylogenetic tree construction. We can see the tree showed that most teosinte lines were basal to the maize lines, while diversity lines were completely separated into two major clusters according to the presence or absence of RegionA (Fig. 5e), which means the NOR flanking region and RegionA as a whole, showed a distinct evolutionary relationship from the entire genome.

RegionA is present in 5 of 17 (29\%) teosinte, indicating it is a standing genetic variant in teosinte. But this frequency rose to $11 / 23(48 \%)$ and 50/60 (83\%) in landrace and maize inbred line respectively, exhibiting a progressively increasing enrichment along with the maize domestication and improvement (Fig. 1f). To examine whether RegionA has been under selection during domestication or modern breeding, a series analysis was conducted. For Tajima's $D$, there is an excess of negative values in RegionA for maize landraces and inbreds $(-0.9695,-1.7032$, respectively, Fig. $5 \mathrm{c})$, which suggests an overall excess of low-frequency mutations. Though the negative value was interpreted as evidence for positive selection, the demographic factors, background selection, or a combination of these factors can also affect the results. Here we scanned the signatures of selective sweeps for RegionA-presence maize landraces and inbreds, using a site frequency spectra (SFS)-based method by SweeD [74]; the composite likelihood ratio (CLR) was calculated with grid sizes of $50 \mathrm{~kb}$ across each chromosome. We then 


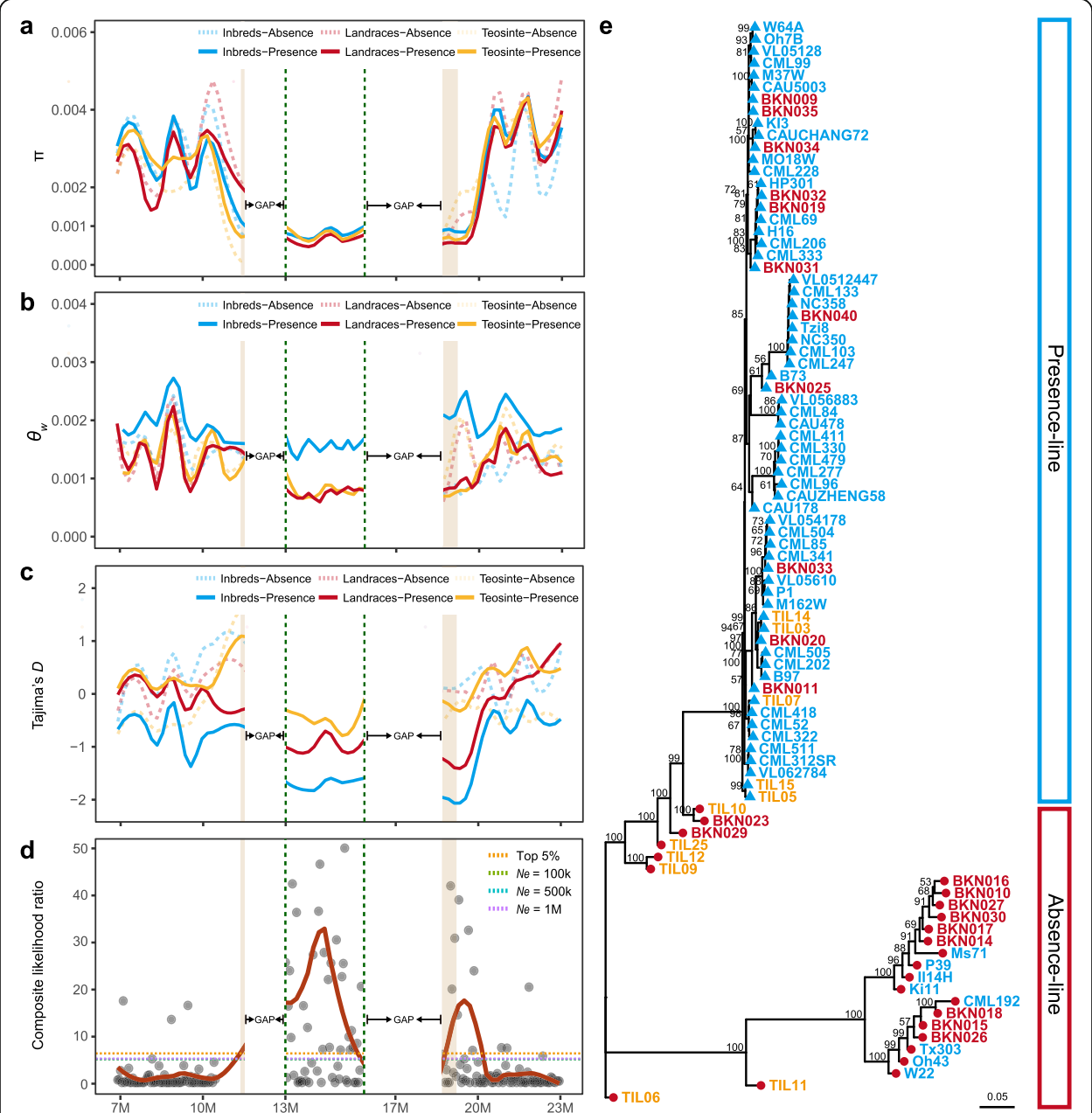

Fig. 5 Genetic diversity of RegionA in the context of domestication. a-d RegionA harbors low genetic diversity and positive selection signals inside. Four indicators of sequence diversity and selection signals, nucleotide diversity $(\pi)$, Watterson's estimator $\left(\theta_{W}\right)$, Tajima's $D$, and composite likelihood ratio (CLR) of modern inbreds were shown in $\mathbf{a}, \mathbf{b}, \mathbf{c}$, and $\mathbf{d}$, respectively. The $100 \mathrm{~kb}$ upstream and $500 \mathrm{~kb}$ downstream flanking regions were indicated by brown blocks. The two green vertical lines indicate RegionA, and indicators were calculated only based on presence line; for $\mathbf{d}$, dashed horizontal lines indicate the cutoff of composite likelihood ratio. e Neighbor-joining tree of the HapMap lines in flanking regions of NOR (upstream $100 \mathrm{~kb}$ and downstream $500 \mathrm{~kb}$ ). The bootstrap values on the tree are based on 1000 replicates and values more than 50 are displayed. Taxa in the tree are represented by different text colors: teosintes (yellow), landraces (red), and improved lines (blue). The tree tip points labeled by blue triangles indicate the RegionA-presence lines, while red circles indicate the absence lines

determined the significance threshold to reject the null model of neutrality based on the demography of maize domestication by coalescent simulations [75]. Finally, we found the significant signature of selection inside the RegionA for landrace and inbred (Fig. 5d, Additional file 1: Figure S10e-g), which rejected the null model of neutrality. Taken together, all the above evidence provided the support that RegionA was targeted by positive selection.

\section{RegionA genes show presence-absence expression patterns}

As a consequence of the PAVs between the inbred lines, the reciprocal hybrids have only haploid allele from the presence parent, in contrast to the diploid genome 
background with both parental alleles, leading to a scenario of segmental dosage. We investigated the transcription of genes located within RegionA using publicly available transcriptome data generated from the seedling shoot (diploid cells) and endosperm (triploid cells) tissue of B73, Mo17, and their reciprocal hybrids (Additional file 2: Table S4) [76-80]. RegionA genes tended to show approximately a 50\% expression level in $\mathrm{BM}$ or $\mathrm{MB}$ hybrid relative to $\mathrm{B} 73$ in the diploid tissue types (Additional file 1: Figure S11a-c). In the endosperm tissue, the majority of the RegionA genes showed a fold change of $\log _{2}(2 / 3)$ when B73 as the maternal parent, and $\log _{2}(1 / 3)$ when B73 as the paternal parent. The change of these RegionA genes was linearly consistent with the dosage ratio asymmetrically contributed by B73 to this triploid tissue (Additional file 1: Figure S11d-f). We also examined the epigenetic modification on these PAV genes, including the positively transcriptional marker H3K4me3, H3K36me3, and H3K9ac [77, 79]. We found that the reduction of modifications was closely correlated with the dosage of transcription activity (Additional file 1: Figure S12).

It is worth noting that there are a number of non-additive B73-specific PAV genes in RegionA (19/43), which showed extremely low or high dosage effects in at least one tissue. These RegionA non-additive genes generally showed a high tissue specificity (Additional file 1: Figure S13), and their proportion relative to the additive genes showed mild but significant differences in some tissues compared with that of the genome-wide level (Additional file 2: Table S5). Given the non-additive effect has been acknowledged as a result of the genetic interaction and dosage compensation [81-83], the annotation of these genes implied the potential roles of these RegionA genes in diverse biological processes, such as development, stress response, and chromatin interaction. (Additional file 2: Table S5).

\section{RegionA genes are associated with environmental fitness}

Since the megabase-scale PAV RegionA appeared under strong selection, we explored the underlying function for the local genes, which may serve the biological foundation of the selection. However, compared to a genome-wide level, RegionA significantly enriched more genes that lack Gene Ontology (GO) annotation (Additional file 2: Table S7). In addition, approximately $40 \%$ of the genes in RegionA had no ortholog found in the species outside the Tripsacinae subtribe (Additional file 2: Table S7), obscuring our understanding of the functions of these genes.

We designed a different approach to explore the possible function of RegionA genes by examining the transcriptome changes affected by RegionA. From B73-Mo17 NearIsogenic Lines [84], we selected two NILs, and referred to as $B^{M}$ for a line with Mo17 RegionA loci introgressed into B73-like background, and as $\mathrm{M}^{\mathrm{B}}$ for a line vice versa (Additional file 1: Figure S14a,b). Transcriptome analysis was conducted for both NILs, as well as the B73 and Mo17 parental inbreds as controls. The presence or absence of RegionA resulted in only a subtle change in the genome-wide context, as the NILs showed a highly similar transcriptome as their respective parental inbred (Additional file 1: Figure S14c). Nevertheless, a conspicuous proportion of the transcriptome, 2592 genes and 2151 genes, respectively, was identified as differentially expressed genes (DEGs) in $\mathrm{B}^{\mathrm{M}}$ and $\mathrm{M}^{\mathrm{B}}$ compared to the corresponding inbred background, respectively (Additional file 1: Figure S15a-c). To evaluate the effect of RegionA on the global 
transcriptome, DEGs were filtered according to three criteria: (1) the DEGs located within the RegionA were excluded; (2) to rule out the interference from the imperfect purity of the genetic background of the NILs, we filtered out the DEGs that contain Mo17 SNPs in $\mathrm{B}^{\mathrm{M}}$ or $\mathrm{B} 73$ SNPs in $\mathrm{M}^{\mathrm{B}}$ beyond RegionA, as well as the target genes of B73/Mo17 eQTLs [85] which overlapped with SNPs among the NILs used in this study (see "Methods"); (3) only the overlapping up- or downregulated DEGs between $\mathrm{B}^{\mathrm{M}}$ and $\mathrm{M}^{\mathrm{B}}$ datasets were retained. A total of 473 highly confident DEGs putatively modulated by RegionA were obtained, including 255 genes that downregulated in $\mathrm{B}^{\mathrm{M}}$ and upregulated in $\mathrm{M}^{\mathrm{B}}, 143$ genes that upregulated in $\mathrm{B}^{\mathrm{M}}$ and downregulated in $\mathrm{M}^{\mathrm{B}}$ (Additional file 1: Figure S15d). GO enrichment analysis of these DEGs revealed that they were mainly enriched in transcription and metabolic regulation response to stress, in particular, to temperature stress response (Additional file 1: Figure S15e). We further analyzed public maize landrace data from Meso- and South America (Additional file 2: Table S8) [39]. In agreement with the putative role of temperature response, we found that RegionA-absence lines predominantly enriched in the highland environment, where the general temperature is cooler (Additional file 1: Figure S16). Indeed, a significant association between RegionA and altitude was detected (F-test, $P$ value $=0.0219$, Additional file 2: Table S9). To test whether RegionA might be related to some agronomic traits, we performed association analysis between RegionA and phenotypes using a previously reported association panel [86]; our results suggest that RegionA was significantly associated with heading date and ear height $(P$ value $=6.12 \mathrm{E}-04,4.59 \mathrm{E}-04$, respectively). In summary, these results suggest that the evolution adaptation of Zea family to various environments may be attributed to the phenotypic consequence caused by the megabase-scale structure variation of the RegionA.

\section{Discussion}

\section{Recombination repression contributed to the preservation of large PAVs}

Despite the growing evidence supporting the role of PAVs in the intraspecific genome evolution, megabase-scale PAVs have been rarely characterized in plant species including maize. It had been difficult to detect those large PAVs possibly due to the lack of long-read sequencing data until recently, but another major reason is that large PAVinduced heterozygosity in an intraspecific hybrid could be progressively shuffled by recombination during evolution [16, 20].

The fascinating intactness of the RegionA, which is highly conserved in the presence lines versus almost entirely missing in absence lines, capacitates the detection of megabase-scale PAVs as survival from evolution erosion. A strong negative association between transposable elements (TEs) and meiotic recombination rates across the genome was confirmed [87-90], while the localization of recombination also tends to show negative correlations with local repetitive-element densities [68,91]. Consistent with that, the pericentromeric location warrants the suppressed recombination around RegionD [68], facilitating the preservation of this large PAV from convergence. RegionA, located on the distal chromosome arm end, seemed to challenge the above hypothesis (Fig. 4). This paradox was reconciled by our later finding that RegionA, located within the NOR, is flanked by rDNA. It has been found few meiotic DSBs occur in the rDNA in yeast [91]. A number of meiotic DSBs within the rDNA were reported 
in maize [69]; however, due to an underestimate of the abundance of rDNA copies used in their calculations, meiotic DSBs in the rDNA may actually be quite infrequent. Furthermore, suppressed recombination rate in the rDNA region has been justified [68, 70], suggesting the occasional DSBs in the rDNA tend to be repaired by any other mechanism but homologous recombination. A recent study in Arabidopsis confirmed the model that highly repetitive $45 \mathrm{~S}$ rDNA arrays are protected from SPO11-induced meiotic DSBs as well as the meiotic recombination machinery by their recruitment into the nucleolus [92]. Our finding on the RegionA within the NOR highlighted the consequence of the diminished recombination, which led to the largest PAV found in maize showing highly conserved sequence as a "fossil" in the long term of evolution (Figs. 3 and 4). The enrichment of repeat sequences in the recombination coldspots explained the difficulty in the assembly of RegionA flanking rDNA region (Additional file 1: Figure S1c,d). This also implies that large PAVs may be underestimated, considering that most genome assemblies nowadays are not based on long-read technologies.

\section{Ancient "fossil" PAV unveils the Tripsacum segment migration into Zea}

Nowadays overwhelming evidence from genomics and molecular biological analysis authentically proved maize domestication from its wild ancestor teosinte. However, the previous process, speciation of Zea, remains to be elucidated. Along with the speciation raised by interspecific hybridization, a simultaneous effect is that the homologous recombination and introgression between ancestral species also generate PAV in the genome [29-31]. Thanks to the integrity of the RegionA protected by the NOR, providing the opportunity to trace the footprint may be preserved against the evolution dynamic. RegionA was found conservatively shared among Tripsacum and Zea, but not detectable in more distant sister species, suggesting a close involvement of Tripsacum in the Zea evolution. Our in-depth analysis revealed the drastically reduced absolute genetic divergence between Tripsacum and Zea at RegionA compared with other loci in the genome (Additional file 1: Figure S9), which was generally considered as the sign of recent gene flow distinguish from shared ancestral variation [66, 67]. We also found evidence supporting gene migration from Tripsacum to teosinte and maize landrace (Fig. 2), raising our postulation that the ancient Tripsacum might contribute to Zea speciation. The flow signal could be only detected from RegionA but not any other four large PAVs reported in this study or any location from the genome. In addition to teosinte parviglumis, RegionA is also stably present in other Zea genus as detected by oligo-FISH in Zea nicaraguensis, Zea luxurians, Zea mays ssp. huehuetenangensis, and Zea mays ssp. mexicana. In all tested Zea samples, the location of RegionA appeared overlapped with the NOR signal, indicating the segment migration occurred no later than the divergence within Zea family (Additional file 1: Figure S17). Overall, our data proposed two possible scenarios for the RegionA origin: RegionA originated from the common ancestor of Tripsacum and Zea; or a direct introgression occurred from speciated ancient Tripsacum to speciating Zea when there was no intercross barrier between yet (Fig. 6). Nevertheless, in the former scenario, as NOR preserves the maize RegionA, factors underpinning the sequence conservation in Tripsacum are also warranted. However, the same strategy on recombination suppression by NOR can be ruled out in Tripsacum since the homologous RegionA localizes separately with NOR 
(Additional file 1: Figure S17). In contrast, the latter scenario seems more promising. This is not merely because of the more recent timing in favor of less variation, but more importantly, RegionA was enriched with Tripsacum-specific LTR retrotransposons, suggesting the introgression happened after the Tripsacum-type LTR retrotransposons were specifically expanded in this genus.

\section{RegionA is associated with environmental adaptation in the maize adaptation during domestication}

In animals, intraspecific genomic diversity is generally thought to derive from relatively small-size variants, resulting from the selection pressure for genome integrity and biological function that otherwise could be dampened by large-size structural variations. Large-size PAVs have been occasionally described in plants, but the number of genes within them is usually limited [32, 33, 93-98]. A surprising fact of our finding here is that 70 genes are located within RegionA, and this is comparable to the average gene density in the whole genome (21.5 genes per megabase compared to a genome-wide average of 18.7 genes per megabase) [52]. In addition, these genes are actively expressed, with a dosage effect between presence and absence lines, linking a possible phenotypic variation to be further investigated as an inset understanding of the heterosis.

Since unbalanced PAVs are present only in some individuals, they have been referred to as dispensable elements generally unnecessary for survival. However, growing evidence supports their roles in various biological processes in plants, arguing that genes within PAVs may be significant in special conditions or particular individuals [16]. Functions of abiotic/biotic response to environmental stresses have been found in PAVs from multiple plant species, and traits of stress response were complementary between different genomes [16, 33, 93-95, 97-100]. More specifically, similar examples of maize SVs have been found to be causative to diverse phenotypes [101-103]. Indeed, a $14 \mathrm{Mb}$ large inversion (Inv4m) was previously identified as introgression from mexicana to high elevation landraces, which is also associated with highland environment adaptation and earlier flowering times in highland maize [103]. Consistent with that, we found the transcriptome effect from the largest B73/Mo17 PAV was enriched in GO categories of the stress response, particularly related to the temperature responding pathway (Additional file 1: Figure S15e). We also observed that RegionA is predominantly absent from the highland landraces (Additional file 1: Figure S16d), implying the role of RegionA functions to modulate the adaptive growth in the tropical lowland, whereas being a fitness cost for the cold environment individuals in the highland.

It is worth noting that the RegionA regulatory function on heat response may not be the only cause for the strong selection signal (Fig. 5), since genes attributed to beneficial agriculture traits could also be the selection targets. Our association analysis suggests that RegionA was related to heading date and ear height. Moreover, a major QTL for grain yield (GY) has been recently reported accounting for $13 \%$ of the variance in GY, this $17 \mathrm{Mb}$ QTL interval coincided with RegionA, and Mo17 allele as one of the founders for the mapping population contribute to low GY values [104]. Another RegionA located gene, $S c m v 1$, encoding atypical h-type thioredoxin, has been identified to impart strong resistance to Sugarcane mosaic virus (SCMV) [105]. In addition, a 


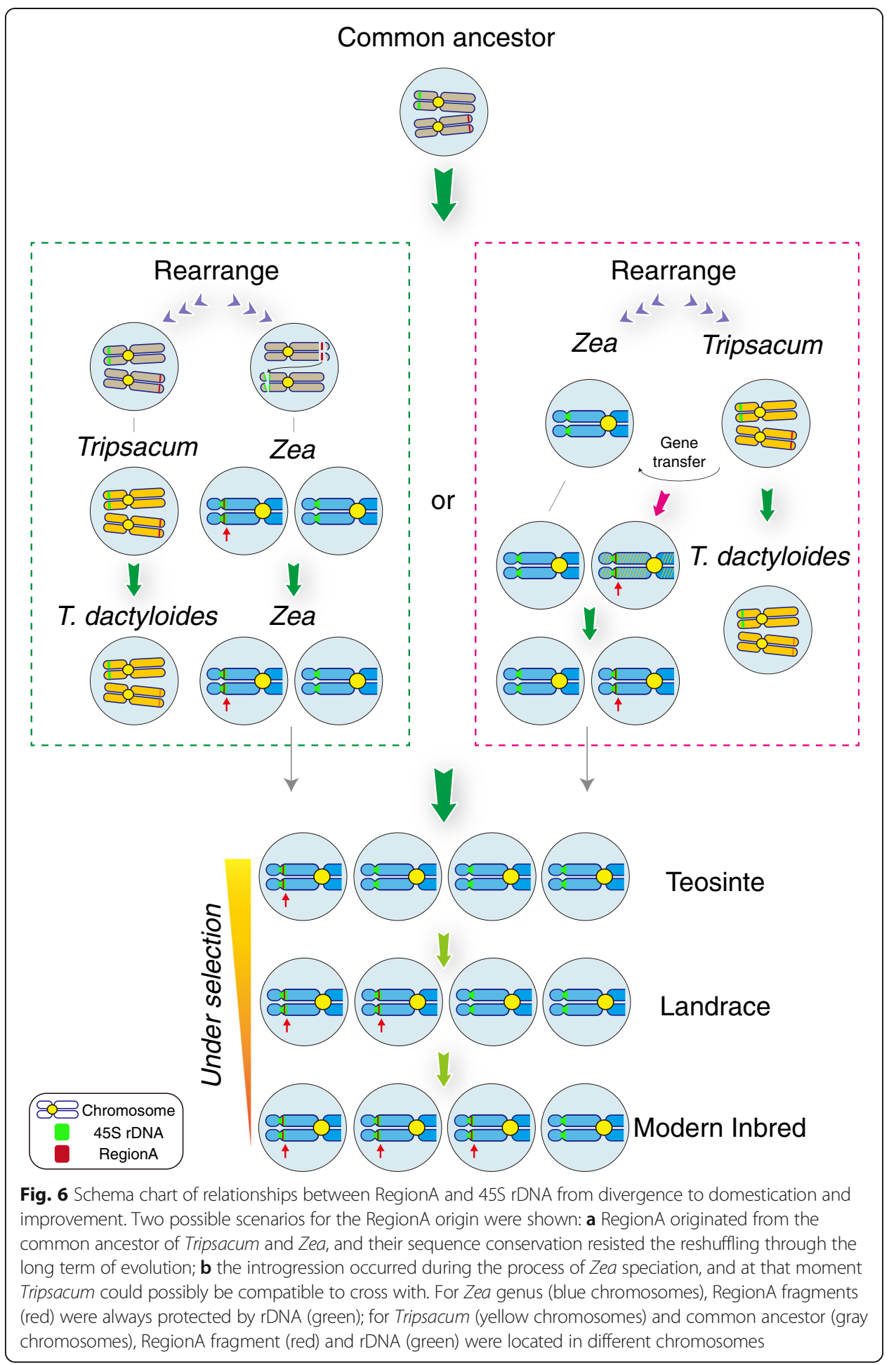

significant SNP for maize upper leaf angle was found located in RegionA [106]. These data suggest the Tripsacum-originated regions indeed play critical roles in genome evolution of Zea, as well as the complex interplay between the genome and the environment.

Intriguingly, the contribution of archaic introgression to modern maize is not unique in plants, but there has been increasing evidence showing that introgression from 
Neanderthals and Denisovans to the human ancestors was subject to selection for the genetic architecture of adaptive traits [107-110]. In general, the introgression from $\mathrm{Ne}$ anderthals functions as deleterious variations thus have been under negative selection during the evolution $[107,111]$. In contrast, a hypoxia pathway gene, EPAS1, was previously identified with positive selection in Tibetans for the altitude adaptation. The EPAS1 haplotype from Tibetans, associated with optimal hemoglobin concentration for high altitude, was found to be introduced by an admixture from Denisovan $[110,112]$. Together with these findings, our study highlights a convergent approach during the genome evolution between plant and human, resulting from the adaptive nature of archaic introgression.

\section{Conclusions}

In this study, a 3.2-Mb PAV element was characterized with high sequence integrity in maize diversity populations, and the embedded location in the nucleolus organizer region (NOR) was associated with the suppressed recombination and preserved the sequence footprint against the evolutionary variation. A series of analyses reveal the striking genomic footprints from Tripsacum in the maize genome, unveiling the origin of this PAV of Zea genus from Tripsacum. We also found this Tripascum-originated segment was under selection during the Zea domestication and adaptation, which is consistent with its role for environmental fitness and agronomic traits. In a general perspective, our results instantiates a strategy for evolution and function analysis of the "fossil" structure variations during genome evolution and speciation, providing a neglected view by the published genome-wide PAV studies.

\section{Methods}

Plant materials

For transcriptome sequencing, the original $B^{M}$ (B73-NIL) and $M^{B}$ (Mo17-NIL) lines MBNIL_b131 and MBNIL_m065 developed by Eichten et al. [84] were requested from Maize Genetics Cooperation Stock Center, backcrossed with B73 and Mo17 respectively for two generations, followed by one round of self-pollination. Then, homozygous RegionA-absence seeds were selected as $\mathrm{B}^{\mathrm{M}}$, and homozygous RegionA-presence seeds were selected as $\mathrm{M}^{\mathrm{B}}$. Accessions of other germplasm used in this study were listed in Additional file 2: Table S10.

\section{Generation of B73 AGPv4.1 genome}

An updated B73 Chr 6 assembly was kindly provided by Dr. Yingping Jiao at Doreen Ware Lab in the Cold Spring Harbor Laboratory. We replaced the original Chr 6 in AGPv4 with this new Chr 6, and named this new genome assembly AGPv4.1. B73 Hi-C data were mapped to the maize genome AGPv4 and v4.1 respectively using Bowtie2 [113], then detected the valid contacts and generated iterative correction matrixes using HiC-Pro v2.10.0 [114], contact matrix was visualized by Juicerbox 1.8.8 [115].

\section{Presence absence region identification}

Raw reads were quality trimmed using Trimmomatic v0.38 [116] with the following parameters (LEADING:3, TRAILING:3, SLIDINGWINDOW:4:15, MINLEN:36). Then 
the trimmed reads were aligned against the B73 AGPv4.1 genome using Bwa mem [117]. Reads with high mapping qualities (MAPQ $\geqq 30$ ) were retained for downstream analysis. Read depth was calculated in 10-kb windows with step size of $1 \mathrm{~kb}$ (at least one base pair overlapped). The windows containing less than 10 reads per genome coverage were defined as absence region.

\section{RegionA genotype identification}

Copy number variation analyses were conducted to identify the copy number of RegionA by cnv-seq [118], using the maize natural variation population (100 HapMap2 line and 99 Meso- and South America landrace) as treats, B73 as a reference, with 100$\mathrm{kb}$ windows. K-means clustering analyses were performed for each line according to their adjusted copy number ratio (CNV ratio) of RegionA windows. When $k=3$, we can easily distinguish three genotypes of RegionA: Homozygous absence $(0 / 0)$, the $\log _{2}$ of CNV ratio ranges from - 2 - 4; Homozygous presence (1/1), the $\log _{2}$ of CNV ratio are approximal 0.6 0.7; Heterozygous (0/1), the $\log _{2}$ of CNV ratio are approximal 0.3 , which showed about half of CNV ratio relative to RegionA-presence homozygous. Although the $\log _{2}$ of $\mathrm{CNV}$ ratio of Homozygous presence (1/1) genotypes were slightly higher than the whole genome ( 0.6 versus 0$)$, we think it may be due to the extremely low genetic diversity of RegionA, so that RegionA contained more reads with highquality alignments than the rest of the genome.

\section{Oligo probe design}

The oligo probe used in this study was designed following a previously published protocol [119]. Briefly, after removing repetitive sequences, RegionA was divided into oligos $(59 \mathrm{nt})$ with a step size of $5 \mathrm{nt}$. Oligos were then mapped to maize genome and those mapped onto ( $>75 \%$ homology over all $59 \mathrm{nt}$ ) multiple loci in the genome were discarded. Next, the melting temperature Tm and hairpin Tm of each oligo sequence were calculated, and oligos with $\mathrm{dTM}>10(\mathrm{dTM}=$ melting temperature - hairpin Tm) were retained. Oligos were synthesized by MYcroarray (Ann Arbor, MI, USA) and labeled with digoxigenin, following a previously published protocol [119]. Oligo probe information is listed in Additional file 2: Table S11.

FISH

Kernels of maize and Zea genus were germinated in a growth chamber for 2-3 days, and $1-2 \mathrm{~cm}$ primary root tips were collected for FISH analysis. Mitotic metaphase and pachytene chromosomes were prepared as described previously [120]. FISH and oligoFISH were performed according to previously published protocols [121, 122] . Briefly, DNA probes were labeled with biotin-11-dUTP (Vector Laboratories) and digoxigenin11-dUTP (Roche) via nick translation. Chromosomes were counterstained with DAPI in VectaShield antifade solution (Vector Laboratories). Biotin- and digoxigenin-labeled probes were detected by anti-biotin fluorescein (Vector Laboratories) and antidigoxigenin rhodamine (Roche), respectively. FISH images were captured with the Olympus BX61 epifluorescence microscope equipped with a CCD camera (QImaging; RETGA-SRV FAST 1394), and processed with Image-Pro Plus 6.0 software (Media Cybernetics). Adobe Photoshop CS3 software was used for final contrast adjustment of 
the images. LTR-RT fragments used for FISH probes were amplified with primers listed in Additional file 2: Table S12.

\section{Estimation of insertion timing for LTR-RTs}

We aligned the 5' LTR and 3' LTR of each intact LTR-RT by MUSCLE v3.8.31 [123], and then used a K2P substitution model to calculate relative ages using the R package APE [124]. We converted these relative ages to absolute time using a mutation rate of $3.3 e-8$ [125].

\section{Footprint tracing of Tripsacum-specific retroelement in maize}

All transposable element annotation were fetched from Gramene [126] : ftp://ftp. gramene.org/pub/gramene/release-62/gff3/zea_mays/repeat_annotation/. RegionA LTR-RTs were re-annotated using previous methods [52] with B73 AGPv4.1 genome sequence. Random regions were generated by Bedtools [127] with function random for retrotransposon family comparison.

To identify the low abundance LTR-RTs in B73, genome-wide LTR-RTs were mapped against the 5'LTR sequences of all the RegionA LTR-RTs using BLASTN with default parameters. The results were then filtered with $80-80-80$ rule [128], by which the elements show at least $80 \%$ similarity using at least 80 base pairs of $80 \%$ of the element's 5'LTR. We calculated the hit number for each RegionA LTR-RTs. LTR-RTs with hit number less than 100 were thought as low abundance LTR-RTs in B73.

To identify the high abundance LTR-RTs in Tripsacum, we extracted the entire element sequence for all the RegionA LTR-RTs, then resequencing data of Tripsacum and Zea luxurians were mapped against these sequences using Bwa [117] with default parameters. To correct the differences of sequencing data depth and length of elements, reads per kilobase per million mapped (RPKM) was calculated for each LTR-RT. If a LTR-RT has a ratio of RPKM $M_{\text {Tripsacum }}$ to $\mathrm{RPKM}_{\text {luxurians }}$ more than 2, and the $\mathrm{RPKM}_{\text {Trip- }}$ sacum was more than 30 but RPKM luxurians $_{\text {was }}$ less than 30 , we thought it as high abundance LTR-RT in Tripsacum.

The intersection of low abundance LTR-RTs in B73 and high abundance LTR-RTs in Tripsacum were designated as Tripsacum-specific enriched LTR-RTs (Tse-LTR-RTs). All genomic sequences of Tripsacum dactyloides clones (TC-25, TC-24, TC-12, TC-5, TFB8-15, TF-B5-2, TF-B5-3) [64] were downloaded from NCBI, then we mapped these sequences against the RegionA 5'LTR sequences from B73 AGPv4.1 using BLASTN with default parameter. After filtering the BLASTN results with the 80-80-80 rule [128], these remaining LTR-RTs were classified as Tripsacum clones consistent LTR-RTs.

B73 annotated CDS sequences in RegionA were extracted, and unique CDS for each gene was achieved by merging the transcripts for the genes with multiple transcript isoforms. Tripsacum resequencing reads were aligned to B73 genome and the percentage of CDS coverage was calculated using in-house Shell scripts. A Tripsacum consistent gene was defined by coverage higher than $70 \%$.

\section{Genetic introgression identification}

Due to the low coverage of the previously accessible resequencing data from Tripsacum, resequencing of three additional Tripsacum lines was conducted, generating 
coverage depth ranging from $11.22 \times$ to $14.48 \times$. In addition to four Tripsacum lines, RegionA-presence lines from the diversity panel were retained for the following analysis, including teosinte $(n=5)$, landrace $(n=11)$, and inbred $(n=50)$. Allele frequencies for each subgroup were estimated using the ANGSD "-doMafs" command [129]. Only polymorphic sites for which $50 \%$ of the individuals in each group have read support were retained. Introgression events among Tripsacum, teosinte, maize landrace, and modern inbred were detected by Treemix [130]. For each number of migrations, the maximum likelihood (ML) tree was generated based on 1000 replicates with a block size of 5000 sites.

We used the absolute sequence divergence $\left(d_{x y}\right)$ to examine genetic differentiation across the genome between Tripsacum and Zea, only RegionA-presence lines were used for calculating, $d_{x y}$ was computed using the script popgenWindows.py (github. com/simonhmartin/genomics_general) with a sliding window of $20 \mathrm{~kb}$ and a step of 10 $\mathrm{kb}$.

\section{Transcriptome and histone modification data analysis}

For the transcriptome analysis of the NIL lines, $\mathrm{B}^{\mathrm{M}}, \mathrm{M}^{\mathrm{B}}, \mathrm{B} 73$, and Mo17 plants were grown in a growth chamber at $28^{\circ} \mathrm{C}$ for $12 \mathrm{~h}$ of light and $25^{\circ} \mathrm{C}$ for $12 \mathrm{~h}$ of darkness for 2 weeks before harvested for RNA extraction. RNA was isolated using a Quick RNA isolation Kit (Huayueyang Biotechnology of Beijing, http://www.huayueyang.com/), followed by mRNA library construction (Novogene) and sequencing at Illumina HiSeq2500 platform.

RNA-seq reads were mapped to B73 AGPv4 genome by HISAT2 [131]. Cufflinks v2.2.1 [132] was used to estimate normalized gene expression values (FPKM). Differential expression analysis was carried by Cuffdiff v2.2.1 [133]. Genes with an expression fold change $\geqq 2$ and FDR $\leqq 0.05$ were defined as differentially expressed. Comparison between $\mathrm{B}^{\mathrm{M}}$ and $\mathrm{B} 73$, as well as between $\mathrm{M}^{\mathrm{B}}$ and Mo17, were conducted for the differentially expressed genes (DEGs) of $\mathrm{B}^{\mathrm{M}}$ and $\mathrm{M}^{\mathrm{B}}$. The overlapped DEGs of $\mathrm{B}^{\mathrm{M}}$ and $\mathrm{M}^{\mathrm{B}}$ were used for downstream analysis. We merged bam files of replicates respectively and discarded the low-quality reads $(\mathrm{MAPQ}<20)$, then all samples were conducted jointcalling by samtools mpileup [134] and sites with QUAL $<10$ and DP $<5$ were discarded. The DEGs that contain Mo17 SNPs in $\mathrm{B}^{\mathrm{M}}$ or B73 SNPs in $\mathrm{M}^{\mathrm{B}}$ were filtered out. The B73/Mo17 RIL population eQTLs that overlapped with SNPs between $B^{M}$ and $\mathrm{B} 73$ or $\mathrm{M}^{\mathrm{B}}$ and Mo17 (a 1-kb window centered on top SNPs in the eQTLs) were extracted [85]; the target genes of these overlapped eQTLs were also filtered out. The Singular Enrichment Analysis (SEA) tool in AGRIGO 2.0 [135] was applied for GO enrichment analysis of the final gene set, with default parameters.

The analysis of the transcriptome atlas datasets for tissue types was conducted through the same RNA-seq workflow as described above. We applied a more stringent criterion for identifying B73-specific PAV genes to Mo17. First, we identified a total of 3723 B73 singleton genes using the Genetribe pipeline [136]. Then, we filtered those with more than $30 \%$ CDS regions covered by Mo17 resequencing reads. Finally, we obtained 911 B73-specific PAV genes, 43 of which locate in RegionA.

We calculated the gene expression and made comparisons among B73/Mo17 hybrids and B73 for different tissues, only expressed genes (FPKM $\geqq 1$ in at least one sample) 
were retained. For tissues consisting of diploid cells, B73-specific genes showing a $\log _{2} \mathrm{fc}>0$ in hybrid than B73 were classified as non-additive (higher) genes, while those showing $\log _{2} \mathrm{fc}<-2$ were classified as non-additive (lower) genes. For endosperm (triploid cells), B73-specific genes showing $\log _{2} \mathrm{fc}_{(\mathrm{BM} / \mathrm{B} 73)}>\log _{2}(2 / 3)+1$ or $\log _{2} \mathrm{fc}_{(\mathrm{MB} / \mathrm{B} 73)}>$ $\log _{2}(1 / 3)+1$ were classified as non-additive (higher) genes, while those showing $\log _{2} \mathrm{fC}_{(\mathrm{BM} / \mathrm{B} 73)}<\log _{2}(2 / 3)-1$ or $\log _{2} \mathrm{fc}_{(\mathrm{MB} / \mathrm{B} 73)}<\log _{2}(1 / 3)-1$ were classified as nonadditive (lower) genes.

ChIP-seq data were mapped to the B73 AGPv4 reference genome using Bowtie [137], and the average enrichment levels of transcription start site (TSS) $\pm 2 \mathrm{kbp}$ and transcription termination site (TTS) $\pm 2 \mathrm{kbp}$ for RegionA, RegionB, and RegionC genes $($ FPKM $\geqq 1)$ were calculated respectively.

\section{Data uplifting from AGPv4 to AGPv4.1}

The HapMap3 genotype data [54] and ZeaGBSv2.7 data are available at http:// cbsusrv04.tc.cornell.edu/users/panzea/filegateway.aspx?category=Genotypes. The sequence of upstream $150 \mathrm{bp}$ to downstream $150 \mathrm{bp}$ around these SNPs was extracted and performed BLASTN against the AGPv4.1 genome, only unique alignment of length = 300 with no mismatch were retained. The locus of filtered SNPs was transformed to the new position where these sequences aligned.

\section{Population genetics analysis and selection test}

SNPs were pre-processed for the following analysis, for which, SNPs with a missing rate of more than 50\% were discarded. To construct the phylogenetic tree, we extracted the uplifted HapMap3 SNPs of upstream $100 \mathrm{~kb}$ and downstream $500 \mathrm{~kb}$ of NOR region in chromosome 6, with a missing rate below 0.5 and a minor allele frequency (MAF) above 0.05 among the 100 HapMap lines; for each line, missing rate more than 0.5 was discarded. Finally, total 90 lines were used for the neighbor-joining tree construction by MEGA 7.0.26 [138] under the p-distances model with bootstrapping (1000), and sites with gaps or missing data more than $20 \%$ were deleted. Ggtree [139] was used to visualize the tree.

For nucleotide diversity $(\pi), \theta_{W}[140]$ and Tajima's $D$ [141], we divided the teosintes, landraces, and inbreds into 6 groups (teosintes-presence, teosintes-absence, landracespresence, landraces-absence, inbreds-presence, inbreds-absence) according to RegionA's presence or absence, then we calculated nucleotide diversity $(\pi), \theta_{W}$, and Tajima's $D$ for these groups respectively using Vcftools 0.1 .15 [142] with non-overlapping windows of $10 \mathrm{~kb}$ size.

SweeD [74] was used for detecting complete selective sweeps in the landraces and modern inbreds, and the composite likelihood ratio (CLR) was calculated with grid sizes of $50 \mathrm{~kb}$ across each chromosome. We then determined the significance threshold to reject the null model of neutrality based on the demography of maize domestication [75]. More specifically, this demographic model estimates an ancestral effective population size of $N_{a}$ about 123,000, and maize split from teosinte about 15,000 generations ago with an initial size of about $5 \%$ of the ancestral $N_{\mathrm{a}}$; maize experienced an exponential population growth after its split from teosinte, reaching a final modern effective population size of $2.98 N_{\mathrm{a}}$. The gene flow from teosinte to maize was estimated to be 
$M_{\mathrm{tm}}=1.1 \times 10^{-5} \times N_{\mathrm{a}}$ migrants per generation, from maize to teosinte was estimated to be $M_{\mathrm{mt}}=1.4 \times 10^{-5} \times N_{\mathrm{a}}$ migrants per generation. The mutation rate per year $(\mu)$ used here is $3 \mathrm{e}-8$, assuming no recombination. In fact, the effective population size $\left(N_{\mathrm{e}}\right)$ in maize is difficult to estimate because of rapid demographic change during and post domestication; here, we ran the models with a set of different $N_{\mathrm{e}}$ values (100 k, $500 \mathrm{k}$, and $1 \mathrm{M})$. We used Hudson's ms [147] to perform coalescent simulations and 10,000 1-Mb simulation datasets were generated, and the simulation datasets were used as inputs for SweeD. The highest 99.99th percentile of the CLR distribution of simulation datasets was used as a significance threshold for real data (CLR $=4.971903$, 5.053264 and 5.127507 for $N_{\mathrm{e}}$ values $=100 \mathrm{k}, 500 \mathrm{k}$, and $1 \mathrm{M}$ ). For modern inbreds, which are different from a population at equilibrium, we considered genomic regions with the top 5\% of CLR scores as a significance threshold (CLR $=6.258513)$.

\section{Profiling of the recombination landscape}

Uplifted ZeaGBSv2.7 data were used to identify recombination events. We extracted US-NAM population [68] GBS data that consists of 5000 F6 RILs from raw data, and divided them according to 25 diverse inbred founders. First, we converted SNPs of HapMap format to abh format using Tassel 5.2.40 [143], which filtered the missing loci, heterozygous loci, and sites with same genotypes in parents. Then, we constructed binmap with 20 SNP windows and a sliding step of 2 SNPs, in each window, and the genotype was defined by the ratio of two kinds of parent genotypes: if the proportion of one kind of genotype in this window is more than $3 / 4$, this kind of genotype will be called, whereas if none of genotype is more than $3 / 4$, the window will be called heterozygous genotype. Regions with no more than ten continuous heterozygous windows were set as breakpoints. We merged the crossover events of presence versus absence (P-vs-A) population and presence versus presence ( $\mathrm{P}$-vs-P) population respectively. For chromosome 6 of AGPv4.1, we calculated the crossovers counts in 1-Mb non-overlapping windows across the whole chromosome. For P-vs-P population, counts in each window were divided by 3786 which is the size of P-vs-P population. While for P-vs-A population, counts in each window were divided by 1198 which is the size of P-vs-A population.

\section{Association analysis of RegionA presence/absence and altitude}

The whole-genome shotgun sequencing data and geographic information were downloaded from Figshare https://figshare.com/articles/GenomeSize_lowcoverage_ Maizedata/5117827 [39]; after discarding extremely low coverage data $(<0.01 \times$ coverage) and non-geographic information data, we fetched a total of 80 landrace accessions. To identify the presence or absence of RegionA, these data were mapped against B73 AGPv4.1 genome by Bwa mem [117], high-quality reads (MAPQ $\geqq 30$ ) were retained, and read depth was calculated in 10-kb windows with step size of $1 \mathrm{~kb}$. For each window, read depth was normalized with the total read number for each sample. Heatmap was generated with $\mathrm{R}$ package pheatmap [144]; all columns were clustered with hierarchical clustering method according to normalized read depth. PCA was performed using Tassel 5.2.40 [143] with default settings. An F-test was used to test the association between RegionA and the altitude, by comparing a full model (altitude $\sim$ PC1 + 
$\mathrm{PC} 2+\mathrm{PC} 3+\mathrm{PC} 4+\mathrm{PC} 5+$ Genotype) and a reduced model (altitude $\sim \mathrm{PC} 1+\mathrm{PC} 2+$

PC3 + PC4 + PC5), with the "anova" and "lm" package in R (Supplemental Table 10).

\section{Association analysis of RegionA presence/absence and agronomic traits}

To test the association between RegionA and agronomic traits, we used the maize association population previously reported [86]; two pairs of primers designed within RegionA were used to determine RegionA presence/absence. Phenotypic data of association population were fetched from http://www.maizego.org/Resources.html; the associations between RegionA and phenotypes were tested using a mixed linear model that corrects for population structure and family relatedness [145] in Tassel 5.2.40 [143].

\section{Supplementary Information}

The online version contains supplementary material available at https://doi.org/10.1186/s13059-021-02448-2.

Additional file 1: Figure S1. Hi-C interaction matrix near representative PAVs with different B73 genome assembly version. Figure S2. Genotype variation and percentage of presence bin among different lines in the other representative B73 PVs. Figure S3. Genotype variation and percentage of presence bin among different lines in the other representative Mo17 PVs. Figure S4. Syntenic dotplot between B73 AGPV4 (Chr6) and genomes of PH207, B104, Mo17, W22, 24 NAM founders and some other outgroup species (rice and setaria). Figure S5. The majority of RegionA genes are either completely present or absent in diversity lines. Figure S6. RegionA genotypes in the diversity panel of maize. Figure S7. Characteristics of RegionA transposable elements. Figure S8. Gene flow detected among Tripsacum and Zea for ( $a-b)$ RegionA when (a) $m=2$ and (b) $m=3$; for (c-e) maize whole chromosome 6 when (c) $m=1,(d) m=2$ and (e) $m=3$. Figure S9. Absolute sequence divergence $\left(d_{x y}\right)$ between Tripsacum and Zea. Figure S10. Genetic diversity and selection signals among different populations. Figure S11. Transcription profiling of the genes in PAVs by RNA-seq for (a-c) diploid cells and ( $d-f)$ triploid cells. Figure S12. Histone modification change with PAVs genes in (a-i) seedling shoot (diploid cells) and (j-m) 12 DAP endosperm (triploid cells) dissected from B73, Mo17 and the reciprocal hybrids. Figure S13. Venn diagram of intersections of non-additive RegionA genes in different tissues. Figure S14. Schematic diagram shows genotype of the B73-Mo17 NearIsogenic Lines (NILs) and their parental inbreds selected for transcriptome sequencing. Figure S15. Transcriptomic response resulting from the RegionA PAV on the genome background. Figure S16. Presence or absence of RegionA was significantly associated with altitude of maize landrace. Figure S17. FISH analysis of the relative localization of the RegionA (red signal, marked as Oligo) and 45S rDNA (green signal, marked as 45S) on metaphase chromosomes of Zea genus and Tripsacum.

Additional file 2: Table S1. Summary of representative PAVs in B73 and Mo17 genome studied in this research. Table S2. List of lines used to characterize PAVs in this study. Table S3. Tripsacum-specific LTR-RTs in RegionA. Table S4. Collection of data used to examine the dosage effect of the B73/Mo17 PAVs. Table S5. Number of RegionA genes showing non-additive mode. Table S6. Details of non-additive RegionA genes. Table S7. Enrichment analysis of RegionA genes. Table S8. Geographic information of the landraces used in this study. Table S9. Association between the presence/absence of RegionA and altitude, tested by an F-test. Table S10. A summary of plant samples. Table S11. RegionA-specific oligo FISH probe used in this study. Table S12. Primers used in this study.

Additional file 3. Review history.

\section{Acknowledgements}

We thank Qilin Tang (Sichuan Agricultural University) for providing T. dactyloides $(2 n=72$, DL) materials. We thank Tao Zhang (Yangzhou University) for designing oligo probes. We also thank Junpeng Shi (China Agricultural University) for his instructive discussion.

Review history

The review history is available as Additional file 3.

Peer review information

Wenjing She was the primary editor of this article and managed its editorial process and peer review in collaboration with the rest of the editorial team.

\section{Authors' contributions}

Z.D. and W.J. conceived and designed the project. Y.H., W.H., and Y.L. collected the plant materials and generated the sequencing data. Y.H. performed the bioinformatic analysis. W.H., Z.M., G.T.B., and Y.L. performed FISH analysis. Z.D. and Y.H. wrote the manuscript. W.H., K.W., H.W., J.L., J.J., and W.J. further revised the manuscript. The authors read and approved the final manuscript.

Funding

This work was supported by grants from National Key Research and Development Program of China (2016YFD0102003 to W.J. and 2019 YFA0903904 to W.H.), National Natural Science Foundation of China (92035302 to W.J.), Ministry of 
Education of China (111-2-03 to W.J.), National Science Foundation grant IOS-1444514 to J.J., and Chinese Universities Scientific Fund of China Agricultural University to Z.D.

\title{
Availability of data and materials
}

The genomic resequencing data have been deposited into NCBI BioProject under accession number PRJNA639380 [146]. RNA-seq can be downloaded from NCBI SRA database with accession number SRP251136 [147].

\section{Declarations}

Ethics approval and consent to participate

Not applicable.

\section{Consent for publication}

Not applicable.

\section{Competing interests}

The authors declare no competing interests.

\begin{abstract}
Author details
${ }^{1}$ State Key Laboratory of Plant Physiology and Biochemistry, National Maize Improvement Center, Key Laboratory of Crop Heterosis and Utilization (MOE), Joint International Research Laboratory of Crop Molecular Breeding (MOE), China Agricultural University, Beijing 100193, China. ${ }^{2}$ Key Laboratory of Genetics, Breeding and Multiple Utilization of Corps (MOE), Fujian Agriculture and Forestry University, Fuzhou 350002, Fujian, China. ${ }^{3}$ Department of Plant Biology, Department of Horticulture, Michigan State University, East Lansing, MI 48824, USA.
\end{abstract}

Received: 12 March 2021 Accepted: 2 August 2021

Published online: 20 August 2021

\section{References}

1. Messing J, Dooner HK. Organization and variability of the maize genome. Curr Opin Plant Biol. 2006;9:157-63.

2. Buckler ES, Gaut BS, McMullen MD. Molecular and functional diversity of maize. Curr Opin Plant Biol. 2006;9:172-6.

3. Sun S, Zhou Y, Chen J, Shi J, Zhao H, Zhao H, et al. Extensive intraspecific gene order and gene structural variations between Mo17 and other maize genomes. Nat Genet. 2018;50:1289-95.

4. Wellenreuther M, Mérot C, Berdan E, Bernatchez L. Going beyond SNPs: tThe role of structural genomic variants in adaptive evolution and species diversification. Mol Ecol. 2019;28:1203-9.

5. Conrad DF, Hurles ME. The population genetics of structural variation. Nat Genet. Nature Publishing Group. 2007;39:S30-6.

6. Alkan C, Coe BP, Eichler EE. Genome structural variation discovery and genotyping. Nature Reviews Genetics. 2011;36376.

7. Lei L, Goltsman E, Goodstein D, Wu GA, Rokhsar DS, Vogel JP. Plant pan-genomics comes of age. Annu Rev Plant Biol. 2021;72:411-35.

8. Tuzun E, Sharp AJ, Bailey JA, Kaul R, Morrison VA, Pertz LM, et al. Fine-scale structural variation of the human genome. Nat Genet. 2005;37:727-32.

9. Zhou Y, Minio A, Massonnet M, Solares E, LV Y, Beridze T, et al. The population genetics of structural variants in grapevine domestication. Nat Plants. 2019;5:965-79.

10. Kou Y, Liao Y, Toivainen T, LV Y, Tian X, Emerson JJ, et al. Evolutionary genomics of structural variation in Asian rice (Oryza sativa) domestication. Mol Biol Evol. 2020;37:3507-24.

11. Lan R, Reeves PR. Intraspecies variation in bacterial genomes: the need for a species genome concept. Trends Microbiol. 2000;8:396-401.

12. Mclnerney JO, McNally A, O'Connell MJ. Why prokaryotes have pangenomes. Nat Microbiol. 2017;2:17040.

13. Welch RA, Burland V, Plunkett G 3rd, Redford P, Roesch P, Rasko D, et al. Extensive mosaic structure revealed by the complete genome sequence of uropathogenic Escherichia coli. Proc Natl Acad Sci U S A. 2002;99:17020-4.

14. 1000 Genomes Project Consortium, Abecasis GR, Altshuler D, Auton A, Brooks LD, Durbin RM, et al. A map of human genome variation from population-scale sequencing. Nature. 2010;467:1061-73.

15. Spielmann M, Lupiáñez DG, Mundlos S. Structural variation in the 3D genome. Nat Rev Genet. 2018;19:453-67.

16. Marroni F, Pinosio S, Morgante M. Structural variation and genome complexity: is dispensable really dispensable? Curr Opin Plant Biol. 2014;18:31-6.

17. Read BA, Kegel J, Klute MJ, Kuo A, Lefebvre SC, Maumus F, et al. Pan genome of the phytoplankton Emiliania underpins its global distribution. Nature. 2013;499:209-13.

18. Yue J-X, Li J, Aigrain L, Hallin J, Persson K, Oliver K, et al. Contrasting evolutionary genome dynamics between domesticated and wild yeasts. Nat Genet. 2017;49:913-24.

19. Alonge $M$, Wang $X$, Benoit $M$, Soyk S, Pereira $L$, Zhang $L$, et al. Major impacts of widespread structural variation on gene expression and crop improvement in tomato. Cell. 2020;182:145-61.e23.

20. Bayer PE, Golicz AA, Scheben A, Batley J, Edwards D. Plant pan-genomes are the new reference. Nat Plants. 2020;6:914-20.

21. Cai X, XU SS. Meiosis-driven genome variation in plants. Curr Genomics. 2007:8:151-61.

22. Bennetzen $J \mathrm{~L}$, Wang $H$. The contributions of transposable elements to the structure, function, and evolution of plant genomes. Annu Rev Plant Biol. 2014;65:505-30.

23. Liu S, Ying K, Yeh C-T, Yang J, Swanson-Wagner R, Wu W, et al. Changes in genome content generated via segregation of non-allelic homologs. Plant J. 2012. p. 390-9.

24. Feurtey A, Stukenbrock EH. Interspecific gene exchange as a driver of adaptive evolution in fungi. Annu Rev Microbiol. 2018;72:377-98. 
25. Dunning LT, Olofsson JK, Parisod C, Choudhury RR, Moreno-Villena JJ, Yang Y, et al. Lateral transfers of large DNA fragments spread functional genes among grasses. Proc Natl Acad Sci U S A. 2019;116:4416-25.

26. Syvanen M. Evolutionary implications of horizontal gene transfer. Annu Rev Genet. 2012;46:341-58.

27. Alix K, Gérard PR, Schwarzacher T, Heslop-Harrison JSP. Polyploidy and interspecific hybridization: partners for adaptation, speciation and evolution in plants. Ann Bot. 2017;120:183-94.

28. Wendel JF, Jackson SA, Meyers BC, Wing RA. Evolution of plant genome architecture. Genome Biol. 2016;17:37.

29. Hurgobin B, Golicz AA, Bayer PE, Chan C-KK, Tirnaz S, Dolatabadian A, et al. Homoeologous exchange is a major cause of gene presence/absence variation in the amphidiploid Brassica napus. Plant Biotechnol J. 2018;16:1265-74.

30. Maccaferri M, Harris NS, Twardziok SO, Pasam RK, Gundlach H, Spannagl M, et al. Durum wheat genome highlights past domestication signatures and future improvement targets. Nat Genet. 2019;51:885-95.

31. International Wheat Genome Sequencing Consortium (IWGSC), IWGSC RefSeq principal investigators:, Appels R, Eversole K, Feuillet $C$, Keller B, et al. Shifting the limits in wheat research and breeding using a fully annotated reference genome. Science. 2018;361.

32. Golicz AA, Bayer PE, Barker GC, Edger PP, Kim H, Martinez PA, et al. The pangenome of an agronomically important crop plant Brassica oleracea. Nat Commun. 2016;7:13390.

33. SchiessI S-V, Katche E, Ihien E, Chawla HS, Mason AS. The role of genomic structural variation in the genetic improvement of polyploid crops. The Crop Journal. 2019;7:127-40.

34. Gaut BS, Seymour DK, Liu Q, Zhou Y. Demography and its effects on genomic variation in crop domestication. Nat Plants. 2018:4:512-20

35. Whitkus R, Doebley J, Lee M. Comparative genome mapping of Sorghum and maize. Genetics. 1992;132:1119-30.

36. Wei F, Coe E, Nelson W, Bharti AK, Engler F, Butler E, et al. Physical and genetic structure of the maize genome reflects its complex evolutionary history. PLoS Genet. 2007;3:e123.

37. Messing J, Bharti AK, Karlowski WM, Gundlach H, Kim HR, Yu Y, et al. Sequence composition and genome organization of maize. Proc Natl Acad Sci U S A. 2004;101:14349-54.

38. Murat F, Xu J-H, Tannier E, Abrouk M, Guilhot N, Pont C, et al. Ancestral grass karyotype reconstruction unravels new mechanisms of genome shuffling as a source of plant evolution. Genome Res. 2010;20:1545-57.

39. Bilinski P, Albert PS, Berg JJ, Birchler JA, Grote MN, Lorant A, et al. Parallel altitudinal clines reveal trends in adaptive evolution of genome size in Zea mays. PLoS Genet. 2018;14:e1007162.

40. Anderson SN, Stitzer MC, Brohammer AB, Zhou P, Noshay JM, O'Connor CH, et al. Transposable elements contribute to dynamic genome content in maize. Plant J. 2019;100:1052-65.

41. Roessler K, Muyle A, Diez CM, Gaut GRJ, Bousios A, Stitzer MC, et al. The genome-wide dynamics of purging during selfing in maize. Nat Plants. 2019;5:980-90.

42. Swanson-Wagner RA, Eichten SR, Kumari S, Tiffin P, Stein JC, Ware D, et al. Pervasive gene content variation and copy number variation in maize and its undomesticated progenitor. Genome Res. 2010;20:1689-99.

43. Wright SI, Bi IV, Schroeder SG, Yamasaki M, Doebley JF, McMullen MD, et al. The effects of artificial selection on the maize genome. Science. 2005;308:1310-4.

44. Doebley JF, Gaut BS, Smith BD. The molecular genetics of crop domestication. Cell. 2006;127:1309-21.

45. van Heerwaarden J, Doebley J, Briggs WH, Glaubitz JC, Goodman MM, de Jesus Sanchez Gonzalez J, et al. Genetic signals of origin, spread, and introgression in a large sample of maize landraces. Proceed National Acad Sci. 2011. p. 1088-92.

46. Mano Y, Omori F, Kindiger B, Takahashi H. A linkage map of maize $\times$ teosinte Zea luxurians and identification of QTLs controlling root aerenchyma formation. Molecular Breeding. 2008. p. 327-37.

47. Bomblies K, Doebley JF. Molecular evolution of FLORICAULA/LEAFY orthologs in the Andropogoneae (Poaceae). Mol Biol Evol. 2005;22:1082-94.

48. Bennetzen J, Buckler E, Chandler V, Doebley J, Dorweiler J, Gaut B, et al. Genetic evidence and the origin of maize. Latin American Antiquity. Cambridge University Press. 2001;12:84-6.

49. Talbert LE, Doebley JF, Larson S, Chandler VL. Tripsacum andersonii is a natural hybrid involving Zea and Tripsacum: molecular evidence. Am J Bot. 1990;77:722-6.

50. Springer NM, Ying K, Fu Y, Ji T, Yeh C-T, Jia Y, et al. Maize inbreds exhibit high levels of copy number variation (CNV) and presence/absence variation (PAV) in genome content. PLoS Genet. 2009;5:e1000734.

51. Beló A, Beatty MK, Hondred D, Fengler KA, Li B, Rafalski A. Allelic genome structural variations in maize detected by array comparative genome hybridization. Theor Appl Genet. 2010;120:355-67.

52. Jiao Y, Peluso P, Shi J, Liang T, Stitzer MC, Wang B, et al. Improved maize reference genome with single-molecule technologies. Nature. 2017;546:524-7.

53. Dong P, Tu X, Chu P-Y, Lü P, Zhu N, Grierson D, et al. 3D Chromatin Architecture of Large Plant Genomes Determined by Local A/B Compartments. Mol Plant. 2017;10:1497-509.

54. Bukowski R, Guo X, Lu Y, Zou C, He B, Rong Z, et al. Construction of the third-generation Zea mays haplotype map. GigaScience. 2018;7(4):1-12

55. Chia J-M, Song C, Bradbury PJ, Costich D, de Leon N, Doebley J, et al. Maize HapMap2 identifies extant variation from a genome in flux. Nat Genet. 2012;44:803-7.

56. Tenaillon MI, Hufford MB, Gaut BS, Ross-Ibarra J. Genome size and transposable element content as determined by high-throughput sequencing in maize and Zea luxurians. Genome Biol Evol. 2011;3:219-29.

57. Zhu Q, Cai Z, Tang Q, Jin W. Repetitive sequence analysis and karyotyping reveal different genome evolution and speciation of diploid and tetraploid Tripsacum dactyloides. Crop J. 2016;4:247-55.

58. McCormick RF, Truong SK, Sreedasyam A, Jenkins J, Shu S, Sims D, et al. The Sorghum bicolor reference genome: improved assembly, gene annotations, a transcriptome atlas, and signatures of genome organization. Plant J. 2018;93:338-54.

59. Hirsch CN, Hirsch CD, Brohammer AB, Bowman MJ, Soifer I, Barad O, et al. Draft assembly of elite inbred line PH207 provides insights into genomic and transcriptome diversity in maize. Plant Cell. 2016;28:270014.

60. Springer NM, Anderson SN, Andorf CM, Ahern KR, Bai F, Barad O, et al. The maize W22 genome provides a foundation for functional genomics and transposon biology. Nat Genet. 2018;50:1282-8. 
61. Hufford MB, Seetharam AS, Woodhouse MR. De novo assembly, annotation, and comparative analysis of 26 diverse maize genomes. bioRxiv. biorxiv.org; 2021; Available from: https://www.biorxiv.org/content/10.1101/2021.01.14.426684 v1.abstract

62. SanMiguel P, Gaut BS, Tikhonov A, Nakajima Y, Bennetzen JL. The paleontology of intergene retrotransposons of maize. Nat Genet. 1998;20:43-5.

63. Ross-Ibarra J, Tenaillon M, Gaut BS. Historical divergence and gene flow in the genus Zea. Genetics. 2009:181:1399-413.

64. Lamb JC, Birchler JA. Retroelement genome painting: cytological visualization of retroelement expansions in the genera Zea and Tripsacum. Genetics. 2006;173:1007-21.

65. Brohammer AB, Kono TJY, Hirsch CN. The Maize Pan-Genome. Compendium of Plant Genomes. 2018. p. 13-29.

66. Smith J, Kronforst MR. Do Heliconius butterfly species exchange mimicry alleles? Biol Lett. 2013;9:20130503.

67. Martin SH, Davey JW, Jiggins CD. Evaluating the use of ABBA-BABA statistics to locate introgressed loci. Mol Biol Evol. 2015;32:244-57.

68. Rodgers-Melnick E, Bradbury PJ, Elshire RJ, Glaubitz JC, Acharya CB, Mitchell SE, et al. Recombination in diverse maize is stable, predictable, and associated with genetic load. Proc Natl Acad Sci U S A. 2015;112:3823-8.

69. He Y, Wang M, Dukowic-Schulze S, Zhou A, Tiang C-L, Shilo S, et al. Genomic features shaping the landscape of meiotic double-strand-break hotspots in maize. Proc Natl Acad Sci U S A. 2017;114:12231-6.

70. Bauer E, Falque M, Walter H, Bauland C, Camisan C, Campo L, et al. Intraspecific variation of recombination rate in maize. Genome Biology. 2013. p. R103.

71. Li L, Arumuganathan K. Physical mapping of $45 S$ and $5 S$ rDNA on maize metaphase and sorted chromosomes by FISH. Hereditas. 2001;134:141-5.

72. He L, Dooner HK. Haplotype structure strongly affects recombination in a maize genetic interval polymorphic for Helitron and retrotransposon insertions. Proc Natl Acad Sci U S A. 2009;106:8410-6.

73. Morgan AP, Gatti DM, Najarian ML, Keane TM, Galante RJ, Pack Al, et al. Structural Variation Shapes the Landscape of Recombination in Mouse. Genetics. 2017;206:603-19.

74. Pavlidis P, Živkovic D, Stamatakis A, Alachiotis N. SweeD: likelihood-based detection of selective sweeps in thousands of genomes. Mol Biol Evol. 2013;30:2224-34.

75. Beissinger TM, Wang L, Crosby K, Durvasula A, Hufford MB, Ross-lbarra J. Recent demography drives changes in linked selection across the maize genome. Nature Plants. 2016;2:16084.

76. Waters AJ, Makarevitch I, Eichten SR, Swanson-Wagner RA, Yeh C-T, Xu W, et al. Parent-of-origin effects on gene expression and DNA methylation in the maize endosperm. Plant Cell. 2011;23:4221-33.

77. He G, Chen B, Wang X, Li X, Li J, He H, et al. Conservation and divergence of transcriptomic and epigenomic variation in maize hybrids. Genome Biol. 2013;14:R57.

78. Chen J, Zeng B, Zhang M, Xie S, Wang G, Hauck A, et al. Dynamic transcriptome landscape of maize embryo and endosperm development. Plant Physiol. 2014;166:252-64.

79. Dong X, Zhang M, Chen J, Peng L, Zhang N, Wang X, et al. Dynamic and Antagonistic Allele-Specific Epigenetic Modifications Controlling the Expression of Imprinted Genes in Maize Endosperm. Mol Plant. 2017;10:442-55.

80. Zhang M, Xie S, Dong X, Zhao X, Zeng B, Chen J, et al. Genome-wide high resolution parental-specific DNA and histone methylation maps uncover patterns of imprinting regulation in maize. Genome Res. 2014;24:167-76.

81. Birchler JA, Veitia RA. Gene balance hypothesis: connecting issues of dosage sensitivity across biological disciplines. Proc Natl Acad Sci U S A. 2012;109:14746-53.

82. Veitia RA, Bottani S, Birchler JA. Gene dosage effects: nonlinearities, genetic interactions, and dosage compensation. Trends Genet. 2013;29:385-93.

83. Dong Z, Yu J, Li H, Huang W, Xu L, Zhao Y, et al. Transcriptional and epigenetic adaptation of maize chromosomes in Oat-Maize addition lines. Nucleic Acids Res. 2018;46:5012-28.

84. Eichten SR, Foerster JM, de Leon N, Kai Y, Yeh C-T, Liu S, et al. B73-Mo17 near-isogenic lines demonstrate dispersed structural variation in maize. Plant Physiol. 2011;156:1679-90.

85. Li L, Petsch K, Shimizu R, Liu S, Xu WW, Ying K, et al. Mendelian and non-Mendelian regulation of gene expression in maize. PLoS Genet. 2013;9:e1003202.

86. Yang N, Lu Y, Yang X, Huang J, Zhou Y, Ali F, et al. Genome wide association studies using a new nonparametric model reveal the genetic architecture of 17 agronomic traits in an enlarged maize association panel. PLoS Genet. 2014;10: e1004573.

87. Bartolomé C, Maside X, Charlesworth B. On the abundance and distribution of transposable elements in the genome of Drosophila melanogaster. Mol Biol Evol. 2002;19:926-37.

88. Daron J, Glover N, Pingault L, Theil S, Jamilloux V, Paux E, et al. Organization and evolution of transposable elements along the bread wheat chromosome 3B. Genome Biol. 2014;15:546.

89. Tian Z, Rizzon C, Du J, Zhu L, Bennetzen JL, Jackson SA, et al. Do genetic recombination and gene density shape the pattern of DNA elimination in rice long terminal repeat retrotransposons?. Genome Research. 2009;2221-30.

90. Jensen-Seaman MI, Furey TS, Payseur BA, Lu Y, Roskin KM, Chen C-F, et al. Comparative recombination rates in the rat, mouse, and human genomes. Genome Res. 2004;14:528-38.

91. Pan J, Sasaki M, Kniewel R, Murakami H, Blitzblau HG, Tischfield SE, et al. A hierarchical combination of factors shapes the genome-wide topography of yeast meiotic recombination initiation. Cell. 2011;144:719-31.

92. Sims J, Copenhaver GP, Schlögelhofer P. Meiotic DNA Repair in the Nucleolus Employs a Non-homologous End Joining Mechanism. Plant Cell. 2019;31(9):2259-75.

93. Zhao Q, Feng Q, Lu H, Li Y, Wang A, Tian Q, et al. Pan-genome analysis highlights the extent of genomic variation in cultivated and wild rice. Nat Genet. 2018;50:278-84.

94. Gordon SP, Contreras-Moreira B, Woods DP, Des Marais DL, Burgess D, Shu S, et al. Extensive gene content variation in the Brachypodium distachyon pan-genome correlates with population structure. Nat Commun. 2017; 8:2184.

95. Michael TP, Jupe F, Bemm F, Motley ST, Sandoval JP, Lanz C, et al. High contiguity Arabidopsis thaliana genome assembly with a single nanopore flow cell. Nat Commun. 2018:9:541.

96. Lye ZN, Purugganan MD. Copy Number Variation in Domestication. Trends Plant Sci. 2019;24:352-65. 
97. Khan AW, Garg V, Roorkiwal M, Golicz AA, Edwards D, Varshney RK. Super-Pangenome by Integrating the Wild Side of a Species for Accelerated Crop Improvement. Trends Plant Sci. 2020;25:148-58.

98. Liu Y, Du H, Li P, Shen Y, Peng H, Liu S, et al. Pan-Genome of Wild and Cultivated Soybeans. Cell. 2020;182(1):162-76.

99. Zhang J, Chen L-L, Xing F, Kudrna DA, Yao W, Copetti D, et al. Extensive sequence divergence between the reference genomes of two elite indica rice varieties Zhenshan 97 and Minghui 63. Proc Natl Acad Sci U S A. 2016;113:E5163-71.

100. Song J-M, Guan Z, Hu J, Guo C, Yang Z, Wang S, et al. Eight high-quality genomes reveal pan-genome architecture and ecotype differentiation of Brassica napus. Nat Plants. 2020;6:34-45.

101. Studer A, Zhao Q, Ross-Ibarra J, Doebley J. Identification of a functional transposon insertion in the maize domestication gene tb1. Nat Genet. 2011;43:1160-3.

102. Han J-J, Jackson D, Martienssen R. Pod corn is caused by rearrangement at the Tunicate1 locus. Plant Cell. 2012;24: 2733-44.

103. Crow T, Ta J, Nojoomi S, Aguilar-Rangel MR, Torres Rodríguez JV, Gates D, et al. Gene regulatory effects of a large chromosomal inversion in highland maize. PLoS Genet. 2020;16:e1009213.

104. Dell'Acqua M, Gatti DM, Pea G, Cattonaro F, Coppens F, Magris G, et al. Genetic properties of the MAGIC maize population: a new platform for high definition QTL mapping in Zea mays. Genome Biol. 2015;16:167.

105. Liu Q, Liu H, Gong Y, Tao Y, Jiang L, Zuo W, et al. An Atypical Thioredoxin Imparts Early Resistance to Sugarcane Mosaic Virus in Maize. Mol Plant. 2017;10:483-97.

106. Tian F, Bradbury PJ, Brown PJ, Hung H, Sun Q, Flint-Garcia S, et al. Genome-wide association study of leaf architecture in the maize nested association mapping population. Nat Genet. 2011;43:159-62.

107. Sankararaman S, Mallick S, Dannemann M, Prüfer K, Kelso J, Pääbo S, et al. The genomic landscape of Neanderthal ancestry in present-day humans. Nature. 2014;507:354-7.

108. McCoy RC, Wakefield J, Akey JM. Impacts of Neanderthal-Introgressed Sequences on the Landscape of Human Gene Expression. Cell. 2017;168:916-27.e12.

109. Skov L, Coll Macià M, Sveinbjörnsson G, Mafessoni F, Lucotte EA, Einarsdóttir MS, et al. The nature of Neanderthal introgression revealed by 27,566 Icelandic genomes. Nature. 2020;582:78-83.

110. Huerta-Sánchez E, Jin X. Asan, Bianba Z, Peter BM, Vinckenbosch N, et al. Altitude adaptation in Tibetans caused by introgression of Denisovan-like DNA. Nature. 2014;512:194-7.

111. Petr M, Pääbo S, Kelso J, Vernot B. Limits of long-term selection against Neandertal introgression. Proc Natl Acad Sci U S A. 2019;116:1639-44.

112. Simonson TS, Yang Y, Huff CD, Yun H, Qin G, Witherspoon DJ, et al. Genetic evidence for high-altitude adaptation in Tibet. Science. 2010;329:72-5.

113. Langmead B, Salzberg SL. Fast gapped-read alignment with Bowtie 2. Nat Methods. 2012;9:357-9.

114. Servant N, Varoquaux N, Lajoie BR, Viara E, Chen C-J, Vert J-P, et al. HiC-Pro: an optimized and flexible pipeline for Hi-C data processing. Genome Biology. 2015;16:259.

115. Durand NC, Robinson JT, Shamim MS, Machol I, Mesirov JP, Lander ES, et al. Juicebox Provides a Visualization System for Hi-C Contact Maps with Unlimited Zoom. Cell Syst. 2016;3:99-101.

116. Bolger AM, Lohse M, Usadel B. Trimmomatic: a flexible trimmer for Illumina sequence data. Bioinformatics. 2014;30: 2114-20.

117. Li H, Durbin R. Fast and accurate short read alignment with Burrows-Wheeler transform. Bioinformatics. 2009;25:1754-60.

118. Xie C, Tammi MT. CNV-seq, a new method to detect copy number variation using high-throughput sequencing. BMC Bioinformatics. 2009;10:80.

119. Han Y, Zhang T, Thammapichai P, Weng Y, Jiang J. Chromosome-Specific Painting in Cucumis Species Using Bulked Oligonucleotides. Genetics. 2015;200:771-9.

120. Huang W, Du Y, Zhao X, Jin W. B chromosome contains active genes and impacts the transcription of A chromosomes in maize (Zea mays L.). BMC Plant Biol. 2016;16:88.

121. Braz GT, He L, Zhao H, Zhang T, Semrau K, Rouillard J-M, et al. Comparative Oligo-FISH Mapping: An Efficient and Powerful Methodology To Reveal Karyotypic and Chromosomal Evolution. Genetics. 2018;208:513-23.

122. Jin W, Lamb JC, Vega JM, Dawe RK, Birchler JA, Jiang J. Molecular and functional dissection of the maize B chromosome centromere. Plant Cell. 2005;17:1412-23.

123. Edgar RC. MUSCLE: multiple sequence alignment with high accuracy and high throughput. Nucleic Acids Res. 2004;32: $1792-7$.

124. Paradis E, Claude J. Strimmer K. APE: Analyses of Phylogenetics and Evolution in R language. Bioinformatics; 2004. p. 289-90

125. Clark RM, Tavaré S, Doebley J. Estimating a Nucleotide Substitution Rate for Maize from Polymorphism at a Major Domestication Locus. Mol Biol Evol. 2005;2304-12.

126. Tello-Ruiz MK, Naithani S, Stein JC, Gupta P, Campbell M, Olson A, et al. Gramene 2018: unifying comparative genomics and pathway resources for plant research. Nucleic Acids Res. 2018;46:D1181-9.

127. Quinlan AR. BEDTools: The Swiss-Army Tool for Genome Feature Analysis. Curr Protoc Bioinformatics. 2014;47:11.12.1-34.

128. Wicker T, Sabot F, Hua-Van A, Bennetzen JL, Capy P, Chalhoub B, et al. A unified classification system for eukaryotic transposable elements. Nat Rev Genet. 2007;8:973-82.

129. Korneliussen TS, Albrechtsen A, Nielsen R. ANGSD: Analysis of Next Generation Sequencing Data. BMC Bioinformatics. 2014;15:356.

130. Pickrell JK, Pritchard JK. Inference of population splits and mixtures from genome-wide allele frequency data. PLoS Genet. 2012;8:e1002967.

131. Kim D, Langmead B, Salzberg SL. HISAT: a fast spliced aligner with low memory requirements. Nat Methods. 2015;12: 357-60.

132. Trapnell C, Williams BA, Pertea G, Mortazavi A, Kwan G, van Baren MJ, et al. Transcript assembly and quantification by RNA-Seq reveals unannotated transcripts and isoform switching during cell differentiation. Nat Biotechnol. 2010;28:5115.

133. Trapnell C, Hendrickson DG, Sauvageau M, Goff L, Rinn JL, Pachter L. Differential analysis of gene regulation at transcript resolution with RNA-seq. Nat Biotechnol. 2013;31:46-53. 
134. Li H, Handsaker B, Wysoker A, Fennell T, Ruan J, Homer N, et al. The Sequence Alignment/Map format and SAMtools. Bioinformatics. 2009:25:2078-9.

135. Tian, Tian T, Liu Y, Yan H, You Q, Yi X, et al. agriGO v2.0: a GO analysis toolkit for the agricultural community, 2017 update. Nucleic Acids Research. 2017;W122-9.

136. Chen Y, Song W, Xie X, Wang Z, Guan P, Peng H, et al. A Collinearity-Incorporating Homology Inference Strategy for Connecting Emerging Assemblies in the Triticeae Tribe as a Pilot Practice in the Plant Pangenomic Era. Mol Plant. 2020; 13:1694-708.

137. Langmead B, Trapnell C, Pop M, Salzberg SL. Ultrafast and memory-efficient alignment of short DNA sequences to the human genome. Genome Biol. 2009;10:R25.

138. Kumar S, Stecher G, Tamura K. MEGA7: Molecular Evolutionary Genetics Analysis Version 7.0 for Bigger Datasets. Mol Biol Evol. 2016;33:1870-4

139. Yu G, Smith DK, Zhu H, Guan Y, Lam TT-Y. ggtree: anrpackage for visualization and annotation of phylogenetic trees with their covariates and other associated data. Methods Ecol Evol. 2017;28-36.

140. Watterson GA. On the number of segregating sites in genetical models without recombination. Theor Popul Biol. 1975; 7:256-76.

141. Tajima F. Statistical method for testing the neutral mutation hypothesis by DNA polymorphism. Genetics. 1989;123:58595.

142. Danecek P, Auton A, Abecasis G, Albers CA, Banks E, DePristo MA, et al. The variant call format and VCFtools. Bioinformatics. 2011;27:2156-8.

143. Bradbury PJ, Zhang Z, Kroon DE, Casstevens TM, Ramdoss Y, Buckler ES. TASSEL: software for association mapping of complex traits in diverse samples. Bioinformatics. 2007;23:2633-5.

144. Kolde, R. pheatmap: Pretty heatmaps. https://CRAN.R-project.org/package=pheatmap

145. Yu J, Pressoir G, Briggs WH, Vroh Bi I, Yamasaki M, Doebley JF, et al. A unified mixed-model method for association mapping that accounts for multiple levels of relatedness. Nat Genet. 2006;38:203-8.

146. Huang Y, Huang W, Meng Z, Braz GT, Li Y, Wang K, Wang H, Lai J, Jiang J, Dong Z, Jin W. Megabase-scale presenceabsence variation with Tripsacum origin was under selection during maize domestication and adaptation. Datasets. NCBI SRA. 2021. Available from: https://www.ncbi.nlm.nih.gov/bioproject/PRJNA639380

147. Huang Y, Huang W, Meng Z, Braz GT, Li Y, Wang K, Wang H, Lai J, Jiang J, Dong Z, Jin W. Megabase-scale presenceabsence variation with Tripsacum origin was under selection during maize domestication and adaptation. Datasets. NCBI SRA. 2021. Available from: https:/trace.ncbi.nIm.nih.gov/Traces/sra/?study=SRP251136.

\section{Publisher's Note}

Springer Nature remains neutral with regard to jurisdictional claims in published maps and institutional affiliations.

\section{Ready to submit your research? Choose BMC and benefit from:}

- fast, convenient online submission

- thorough peer review by experienced researchers in your field

- rapid publication on acceptance

- support for research data, including large and complex data types

- gold Open Access which fosters wider collaboration and increased citations

- maximum visibility for your research: over $100 \mathrm{M}$ website views per year

At $\mathrm{BMC}$, research is always in progress. 